Article

\title{
Recurrent Neural Network-Based Hourly Prediction of Photovoltaic Power Output Using Meteorological Information
}

\author{
Donghun Lee and Kwanho Kim * \\ Department of Industrial and Management Engineering, Incheon National University, 119 Academy-ro, \\ Yeonsu-gu, Incheon 407-772, Korea; dhlee@inu.ac.kr \\ * Correspondence: khokim@inu.ac.kr; Tel.: +82-32-835-8481
}

Received: 8 November 2018; Accepted: 4 December 2018; Published: 10 January 2019

check for updates

\begin{abstract}
Recently, the prediction of photovoltaic (PV) power has become of paramount importance to improve the expected revenue of PV operators and the effective operations of PV facility systems. Additionally, the precise PV power output prediction in an hourly manner enables more sophisticated strategies for PV operators and markets as the electricity price in a renewable energy market is continuously changing. However, the hourly prediction of PV power outputs is considered as a challenging problem due to the dynamic natures of meteorological information not only in a day but also across days. Therefore, in this paper, we suggest three PV power output prediction methods such as artificial neural network (ANN)-, deep neural network (DNN)-, and long and short term memory (LSTM)-based models that are capable to understand the hidden relationships between meteorological information and actual PV power outputs. In particular, the proposed LSTM based model is designed to capture both hourly patterns in a day and seasonal patterns across days. We conducted the experiments by using a real-world dataset. The experimental results show that the proposed ANN based model fails to yield satisfactory results, and the proposed LSTM based model successfully better performs more than $50 \%$ compared to the conventional statistical models in terms of mean absolute error.
\end{abstract}

Keywords: PV power output prediction; long and short term analysis; deep learning; machine learning; data mining; status reasoning; statistical reasoning

\section{Introduction}

Recently, the importance of photovoltaic (PV) power prediction has been rapidly increasing due to the necessity of the clean energy sources harmless to the environment [1]. Since a PV system converts obtained solar power into electricity, PV operators are interested in stable PV power generation [2], and hybrid energy systems, which utilize multiple renewable power sources, have become popular due to their better efficiency $[3,4]$.

The accurate photovoltaic (PV) power output prediction is considered as an essential for maximizing the potential revenues of PV operators due to price fluctuations [5]. In general, as the future price in a renewable energy market is determined by a bidding mechanism between PV operators and renewable market operators, the expected revenue of a PV operator is directly affected by the ability to predict when and how much electricity will be generated in the future [5]. In the meantime, in an electricity market perspective, PV power output prediction is beneficial to improve the effective operation of electrical system for their consumers though approximating the overall level of electricity that is able to be provided by their PV operators [6]. Moreover, as renewable intraday electricity markets that determine prices in an hourly manner becomes popular $[7,8]$, the sophisticated hourly prediction ability of the PV power output is more required. 
By extension, for the more sophisticated decisions of PV operators and the effective operations of electricity markets, the prediction of PV power outputs in a hourly level is crucially necessary [9]. Since the prices of electricity sold in the most electricity markets for renewable energy dynamically change over time according to the expected amount of generated electricity, the ability to predict PV power outputs for each particular hour in a day enables the adaptive adjustment of the transaction volumes with tailored schedules.

Figure 1 shows typical examples of PV power outputs during two consecutive days in summer and winter in South Korea obtained from the PV power system considered in this research. Here, easy and hard seasons divide according to fluctuation levels of the PV power output. For example, as shown Figure 1, more fluctuation of the PV power output occurs in winter compared to summer, meaning it is harder to predict the PV power output in winter. Naturally, PV power output starts to be generated when the sun rises (usually between 6:00 and 19:00), and it tends to increase until the daily solar intensity peaks (usually between 12:00 and 14:00). The highest PV power output is shown between 10:00 and 14:00 in many cases. After the peak time, it then decreases till sunset.
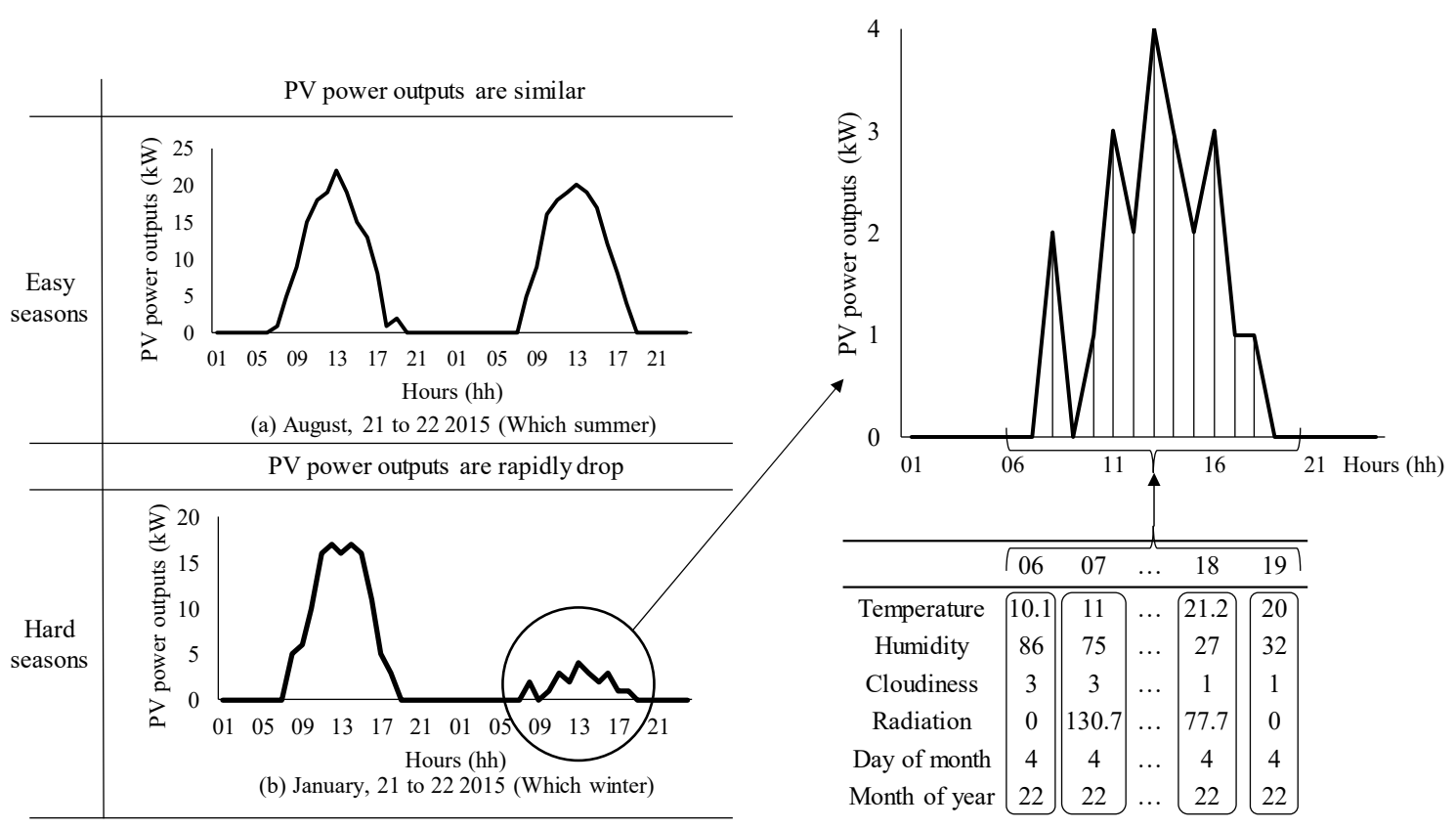

Figure 1. Examples of the PV power output distribution during two consecutive days.

Although it is well known that PV power outputs are strongly related to a variety of meteorological factors such as solar intensity, humidity, temperature, and cloudiness [10], the dynamic changes of such meteorological values make the prediction of PV power outputs significantly challenging. In particular, in the dataset from a PV operation located in Gumi city in South Korea we considered in this study, the average solar intensity and temperature values in a day changed by $34 \%$ and $11 \%$, respectively. Such meteorological changes in a day cause changes of the hourly PV power outputs in the day. For instance, the graph shown in the right in Figure 1 presents an example of a PV power outputs in a day with many significant PV power output changes in a jagged way.

Moreover, in many countries, the hourly patterns of PV power outputs across days also greatly differ with the seasons, which further increases the complexity of the problem. That is, in a particular season, both the specific patterns among hourly PV power outputs in a day and their overall trends across days are considerably different compared to those in other seasons. For instance, as shown in Figure 1, the average changes of hourly PV power outputs across days tend to be much higher (by $16.2 \%$ ) in winter than in summer.

This implies that accurate PV power output prediction is achievable when a prediction model is capable of capturing not only the short term patterns occurring in every hour in a day but also the 
long term patterns across days affected across seasons. That is, on the one hand, since the PV power output for a particular hour in a day is highly associated with those for the previous hours in that day, understanding the hidden patterns over hours from sunrise to sunset is essential from a short term perspective. On the other hand, the PV power outputs during a day are also strongly related with seasonal information such as month of year and day of month from a long term perspective.

As suggested in [11], the prediction models of the PV power output are divided into methods such as regressive, artificial intelligence and remote sensing methods; among them, our models are categorized as intelligent methods. Therefore, based on previous study and prediction ranges, we fall into two categories, such as short-term and long-term predictions as shown in Table 1. Further, in previous studies, the denotation of the short-term was set either 0 to $4 \mathrm{~h}$ in [12] or $24 \mathrm{~h}$ in [13], while the long-term was denoted as one day in [14]. Therefore, considering to the previous studies, in this study the short-term is regarded as under $24 \mathrm{~h}$, and the long-term is considered as $24 \mathrm{~h}$.

Table 1. Overview of previous research related to PV power output prediction.

\begin{tabular}{|c|c|c|}
\hline Categories & Techniques Used & References \\
\hline \multirow{5}{*}{$\begin{array}{l}\text { Short-term } \\
\text { prediction }\end{array}$} & ENN & Dumitru C.-D. et al. [15] \\
\hline & ANN & Izgi, E. et al. [16], Sulaiman S. et al. [17] \\
\hline & RNN & Mellit, A., and Shaari, S. [24], Mandal, P. et al. [25], Abdel-Nasser et al. [26] \\
\hline & DNN & Wang, S. et al. [18], Son, J. et al. [19], Ashraf, I., and Chandra, A. [20] \\
\hline & SVM & Shi, J, et al. [21], Da Silva Fonseca, J. G. et al. [22], Bouzerdoum, M. et al. [23] \\
\hline \multirow{7}{*}{$\begin{array}{l}\text { Long-term } \\
\text { Prediction }\end{array}$} & Hybrid Systems & Chattopadhyay, K et al. [27], Jurasz, J. and Ciapala, B. [28] \\
\hline & ANN & Ding, M. et al. [29], Long, $\mathrm{H}$ et al. [30] \\
\hline & DNN & Jiahao, K. et al. [31], Hiyama, T., and Kitabayashi, K. [32] \\
\hline & PFLRM and RPFLRM & Wang, G. et al. [33] \\
\hline & MAR, LR, ARMAX & Li, Y. et al. [34], Li, Y. et al. [35], Mora-López, L. et al. [36] \\
\hline & Dynamic Neural Network & Al-Messab, N. et al. [37] \\
\hline & RBFNN & Chen, C. et al. [38], Yona, A. et al. [39] \\
\hline
\end{tabular}

There exist short-term PV power output prediction models using simple artificial neural networks (ANN) based on meteorological information [15-17], and deep neural networks by utilizing the solar radiation, module temperature [18,19], and clearness index [20]. Some prediction techniques based on machine learning such as support vector machine (SVM) [21-23], SVM and weather classification methods were proposed [21], support vector regression (SVR) [22] was also proposed, which predicts the PV power output by predicting cloudiness. Much more accurate prediction was reported by considering the time series features such as an adaptive time series model (SARIMA-SVM) [23]. Finally, a recurrent neural network (RNN) model was proposed to capture sequence patterns of PV power outputs [24,25], and the further developed version of RNN, called the long short term memory (LSTM) based model, was proposed [26].

There has been another line of research that aims to predict PV power outputs from a long-term perspective. Hybrid systems incorporating more than one renewable energy source are proposed to predict capacity of electricity generation, using solar and wind power models in [27], and solar and hydro power models in [28]. An ANN using classified weather information based on the past metrological information in [29], and there are other research results that suggest the better performance of DNN-based models with one more hidden layers using weather data [30-32]. Similarity, as an approximation method, the regularized partial functional linear regression model (PFLRM) and multivariate adaptive regression splines (MAR) $[33,34]$ were developed. Further, the ARMAX model and a combination of linear regression (LR) and a machine learning method were proposed [35,36]. For more sophisticated approaches, time series-based models were suggested by utilizing a dynamic neural network [37] and radial basis function neural network (RBFNN) and additionally with weather classification $[38,39]$. However, in the previous research, the RNN model used for PV power prediction is limited to understand long-term dependences among prediction values [40]. In addition, the existing PV power prediction models based on ANN and DNN require additional information such as weather classification information that is unavailable for many areas. 
Therefore, in this paper, we propose a deep learning based approach towards precise hourly prediction of PV power outputs by using meteorological and seasonal factors. For each hour, the proposed approach attempts to capture the relationships between the meteorological factors and the PV power outputs. In addition, unlike the previous approaches, the proposed models consider both the short term relationships among the hourly PV power outputs in a day and their long term relationships across days by combining the well-known deep learning techniques.

Specifically, our approach includes three particular models as follows: First, the proposed single-layer ANN-based model consisting of a single hidden layer attempts to learn PV power outputs in a short term manner without understanding their long term relationships. Second, the suggested multi-layer ANN-based model with multiple hidden layers and the increased number of parameters tries to estimate more accurate PV power outputs than the proposed ANN-based model. Finally, the proposed long and short term memory (LSTM)-based model is designed to understand both short and long term time series patterns, simultaneously, in a single model for further improved predictions.

These models are similar to ones mentioned in previous studies [26,41], but the LSTM- based model proposed in this study has a significant difference because it attempts to estimate hourly PV power output regardless for meteorological conditions and considering meteorological and seasonal trends. In addition, while some models attempt to predict solar radiation [42-44] rather than PV power output prediction, our study focuses on the direct estimation of PV power output.

As shown Figure 2, since the former two models-ANN and DNN-based models-assume that the PV power output in a particular hour is independent of the others, these models predict a single hour PV power by only using the corresponding meteorological and seasonal information for the particular hour. On the contrary, the last one, the LSTM-based model, sequentially utilizes all the hourly features in a day. The LSTM-based model produces the predictions of all the hours in a day at once unlike the ANN-based model. The experiment results using a real world PV power output dataset show that the proposed models can successfully predict PV power outputs compared to the conventional prediction models. In particular, the proposed LSTM model yields the best performance in all of the experimental settings.

\begin{tabular}{|c|c|c|c|c|c|}
\hline \multicolumn{2}{|r|}{ ANN based model } & \multicolumn{2}{|r|}{ DNN based model } & \multicolumn{2}{|r|}{ LSTM based model } \\
\hline Structures & $\begin{array}{l}\text { The three layers with an } \\
\text { input, a singe hidden, } \\
\text { and an output layer }\end{array}$ & Structures & $\begin{array}{l}\text { The nine layers with an } \\
\text { input, a seven hiddens, } \\
\text { and an output layer }\end{array}$ & Structures & $\begin{array}{l}\text { The three layers with an } \\
\text { input, a three recurrent } \\
\text { hiddens, and an output layer }\end{array}$ \\
\hline Predictions & $\begin{array}{l}\text { Dependency among } \\
\text { outputs are not considered }\end{array}$ & Predictions & $\begin{array}{l}\text { Dependency among } \\
\text { outputs are not considered }\end{array}$ & Predictions & $\begin{array}{l}\text { Dependency among } \\
\text { outputs are considered }\end{array}$ \\
\hline
\end{tabular}

Figure 2. Development process of the proposed models.

The paper is composed as follows: in Section 2, we introduce the research framework and the three proposed models to address the considered problem. In Section 3, we evaluate the performances of the proposed models by using a real-world dataset, and we conclude this research in Section 4.

\section{Proposed Methods}

\subsection{Research Framework}

In this paper, we consider the hourly prediction of PV power outputs in a day by using the meteorological and seasonal information given for each hour. In detail, Table 2 summarizes the inputs and the output with the related notations used in this study. For the inputs, four meteorological factors including temperature, humidity, cloudiness, and radiation, and two seasonal factors (month of year and day of month) are considered. 
Table 2. Input and output features and notations for the $i$-th hour in the $j$-th day.

\begin{tabular}{cccc}
\hline & Categories & Features & Notations \\
\hline \multirow{4}{*}{ Inputs } & & Temperature $(t)$ & $t_{i, j}$ \\
& Meteorological factor & Humidity $(k)$ & $k_{i, j}$ \\
& & Cloudiness $(c)$ & $c_{i, j}$ \\
\cline { 2 - 4 } & \multirow{2}{*}{ Seasonal factor } & Radiation $(r)$ & $r_{i, j}$ \\
\hline \multirow{2}{*}{ Output } & & Month of year $(a)$ & $a_{i, j}$ \\
& & Day of month $(b)$ & $b_{i, j}$ \\
\hline & & Predicted & $\hat{s}_{i, j}$ \\
\hline
\end{tabular}

For representing the status of the $i$-th hour in the $j$-th day, a six dimensional input vector, $x_{i, j}=\left\langle t_{i, j}, k_{i, j}, c_{i, j}, r_{i, j}, a_{i, j}, b_{i, j}\right\rangle, i=1, \ldots, n, j=1, \ldots, m$, is defined where $n$ means the number of hours to predict in a day and $m$ is the number of days considered. A set of $n$ input vectors consist of meteorological and seasonal factors for the $j$-th day is used when proposed models repeatedly learn to minimize by calculating between its estimated PV power outputs, denoted as $\hat{s}_{i, j}, i=1, \ldots, n$, and the actual PV power outputs, denoted as $s_{i, j}, i=1, \ldots, n$, to search abstract patterns for accurate prediction of PV power outputs in the $j$-th day. Furthermore, as our models are executable whenever meteorological information is ready, the prediction outputs can be obtained without the limitations on when the predictions are performed.

Figure 3 illustrates how the proposed approach works in terms of the features defined in Table 2. The detailed process of the proposed approach involves two phases. In the training phase, the weights of the prediction models in the proposed framework are updated towards the minimum error between the predicted and actual PV power outputs.

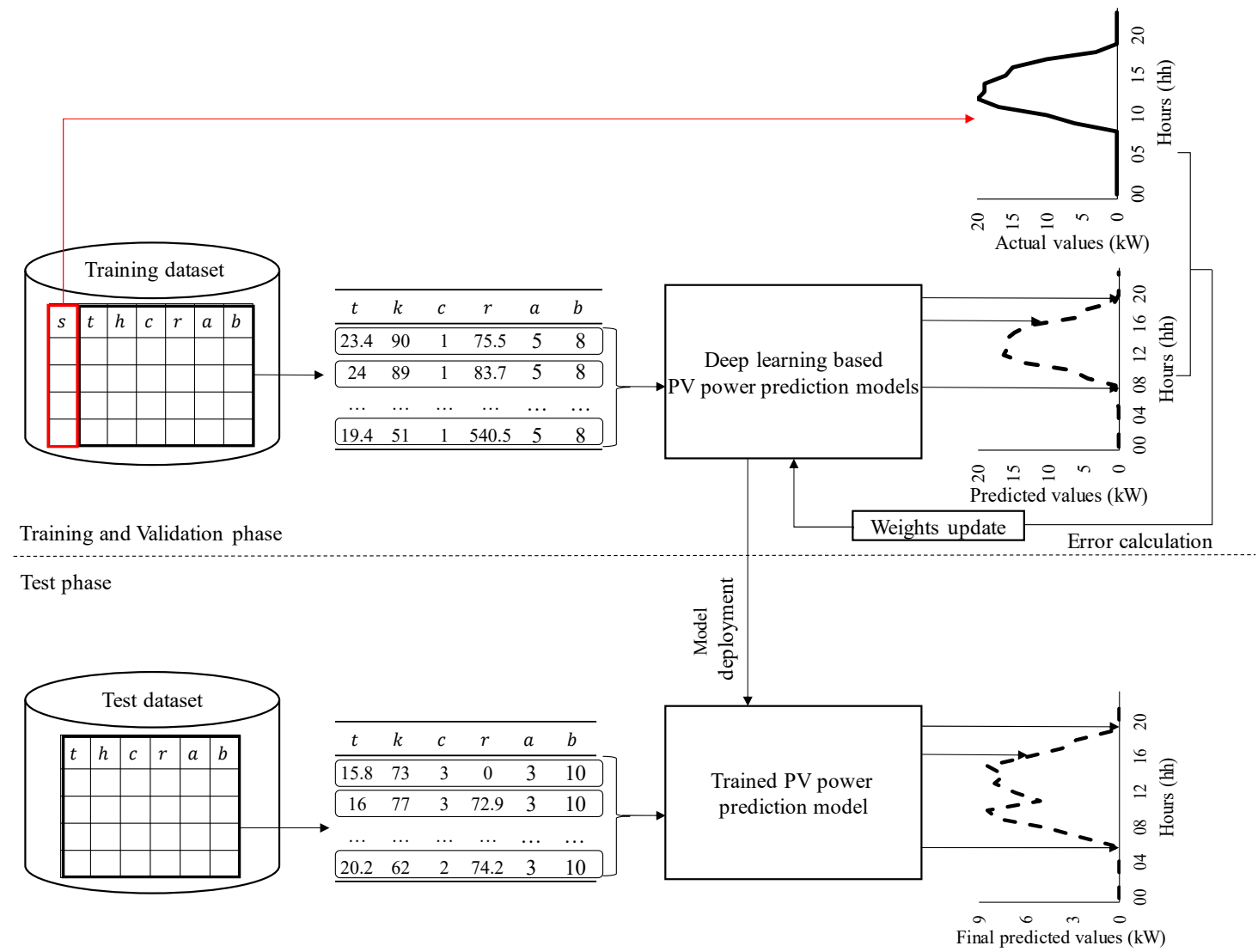

Figure 3. Proposed neural network-based framework for hourly PV power output prediction. 
Our models are to forecast future PV power output by using uncertain meteorological information with many noises. Although the uncertain information contains in historical data, our models attempt to learn hidden patterns that more accurately estimate the PV power output. As we evaluate the performance our models using unseen data, the performance is believed to be generated in real-world settings.

To predict PV power outputs in a daily manner, the proposed models consist of an input layer with $\mathrm{n}$ nodes where each node is for a particular hour, and an output layer with $\mathrm{n}$ nodes where each node is for the prediction of a particular hour corresponding to its input. As this framework attempts to predict the PV power outputs for $14 \mathrm{~h}$ in a day by using the corresponding input factors for each hour. Therefore, we set $\mathrm{n}$ to be 14 in the remainder of this paper. Although the daylight time naturally changes according to seasons, the average daylight times observed in Gumi city in Korea in summer and winter in 2016 were 14 and $12 \mathrm{~h}$, respectively [45]. If these each become longer than 14, one could slightly modify our models by adding an input, a hidden, and an output layer for additional prediction. In the testing phase, by using the weights in the prediction models obtained in the learning phases, the predicted outputs are calculated against the unseen inputs.

\subsection{ANN and DNN Based PV Power Output Prediction}

We propose two types of ANN-based prediction models: single-layer and multi-layer models. Both are widely applied for many prediction problems [46,47], including PV power output predictions $[16,20]$. The single-layer model is well-known for its simple architecture yet quite impressive performances. It consists of a single hidden layer with six nodes, each of which is linearly connected to both inputs and output nodes about input values presented in Table 2. Figure 4a illustrates the network architecture of the proposed single-layer model.

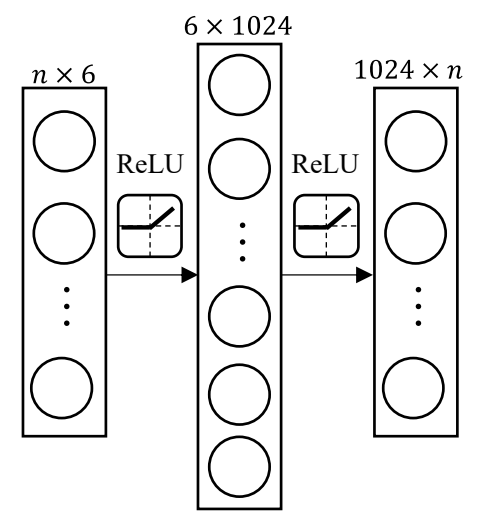

(a) Single-layer model

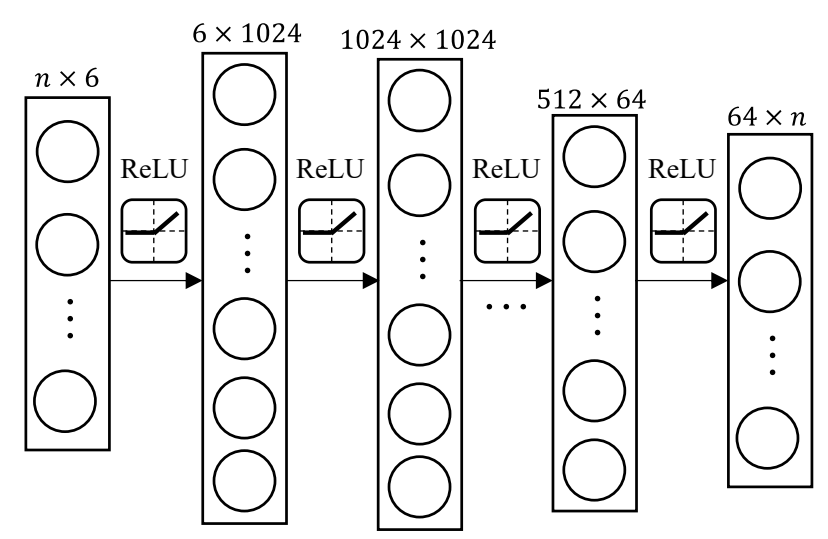

(b) Multi-layer model

Figure 4. Proposed ANN-based models for hourly PV power prediction.

In the proposed ANN-based models, each node in a layer is responsible for producing an output that is utilized as the input values for one or more nodes. By utilizing the weights and the activation function related with a node, the output value is calculated, and the produced output value is then utilized as an input value for the nodes in the next layer.

At first, for a node in the input layer, its input value is to be one of values related with meteorological and seasonal factors, presented in Table 2. For a node in the output layer, based on its input values that come from the nodes in the previous layer, its output value is used as the final prediction for a particular hour based on the position of the node in the output layer.

When a node receives the input values, it calculates the weighted summation of its input values, called the net value for the node. Here, the weight value for each input value is initially set to be a 
random value between zero and one, and it is updated during the training phase that will be discussed later. Specifically, the net value for node $q$, denoted as $n e t_{q}$, is calculated by using Equation (1):

$$
n e t_{q}=\sum_{p=1}^{P} w_{q, p} o_{p}
$$

where $w_{q, p}$ is the weight value corresponding to the connection from node $p$ to node $q, o_{p}$ means the output value produced by node $p$ in the previous layer, and $P$ is the number of nodes connected to node $q$.

By using the net value for node $q$, its output value, denoted as $u_{q}$, is obtained by using Equation (2):

$$
u_{q}= \begin{cases}v\left(\text { net }_{q}\right), & \text { if } \text { net }_{q}>0, \\ 0, & \text { otherwise. }\end{cases}
$$

where $v(\cdot)$ is an activation function that is used to transform an input value into an output value in a non-linear manner.

As the outputs of the nodes in an output layer are considered as the final predicted values of PV power output, $u_{q}, q=1, \ldots, n$. Therefore, for the $j$-th day, $u_{q}$ is considered to be the prediction value for the $q$-th hour in the day, denoted as $\hat{s}_{q, j}$.

Among many popular ones, we adopt the rectified linear unit (ReLU) for all the nodes in the hidden and output layers. ReLU is well-known for providing enhanced training performances and preventing the gradient vanishing and radiant exploding problems [48].

Whenever the proposed model consumes given training instances involving both the meteorological information and the actual PV power output for a particular hour, its weights are gradually updated through an iterative learning process. The back propagation and the Adam optimizer are applied for optimizing the weights of the proposed models $[49,50]$.

For the $j$-th day, the amount of errors between the predicted PV outputs, $\hat{s}_{i, j}, i=1, \ldots, n$, and the actual PV outputs, $s_{i, j}, i=1, \ldots, n$, is measured by means of mean square error (MSE). Based on the MSE values for each training epoch, the Adam optimizer determines how much the weights are needed to be adjusted toward minimizing MSE values for the entire training dataset. For a given training dataset, the MSE based loss value between the actuals and predictions is obtained by using Equation (3):

$$
L=\frac{1}{m} \sum_{j=1}^{m} \frac{\sum_{i=1}^{n}\left(s_{i, j}-\hat{s}_{i, j}\right)^{2}}{n}
$$

In the testing phase, for given input values for each hour, its six dimensional input vector is generated, and then the outputs using Equations (1) and (2) is then simply calculated by using the weights obtained through the training phase.

The second DNN-based model, called the multi-layer model, is the same to the proposed single-layer model except for the fact that it contains seven hidden layers (shown in Figure 4b). The performance difference occurs depending on the number of hidden layers, but we chose seven hidden layers with the best performance through repeated experiments. As an extended version of the first model, it conducts more nonlinear calculations by using more hidden layers before producing the final predictions compared to the first one. The activation function, loss function, and optimizer for this model are the same to those for the first model.

\subsection{LSTM-Based PV Power Output Prediction}

To precisely capture the sequential PV power output patterns hidden in a day and across days, a further improved model, called LSTM-based model, is developed. In details, the proposed model attempts to learn the long-term relationships among the meteorological factors by considering their 
seasonal changes but also to understand the short-term relationships across the PV power outputs in a day.

In particular, when the proposed models are compared in terms of the output, the major difference comes from whether a model is trained the dependency among the consecutive information. That is, the former two models produce output for an hour against the meteorological and seasonal information on hour, the last one results on the output for an hour against the meteorological and seasonal information on the previous hours.

The proposed model consists of a block, called block cell, that collectively determine the intermediate outputs based on the current and past input values by utilizing the long and short term sequential memories, which is borrowed from the basic concept of LSTM [51]. The inputs of a block cell involve the current observations and some parameters while its outputs are a value to present the long-term dependency.

For the $i$-th hour in the $j$-th day, Figure 5 illustrates the architecture of the block cell consisting of three gates such as input, forget, and output gates, denoted as $f_{i, j}, g_{i, j}$, and $o_{i, j}$, respectively. Here, $h_{i, j}$ means the outputs of the block cell in charge of representing the short term pattern for the observation related with the $i$-th hour. Additionally, (i) $b_{g}, b_{f}$, and $b_{o}$ are the bias vectors, and (ii) $W_{h g}, W_{h f}$, and $W_{h o}$ are the weight matrices for the input, forget, and output gates, respectively.

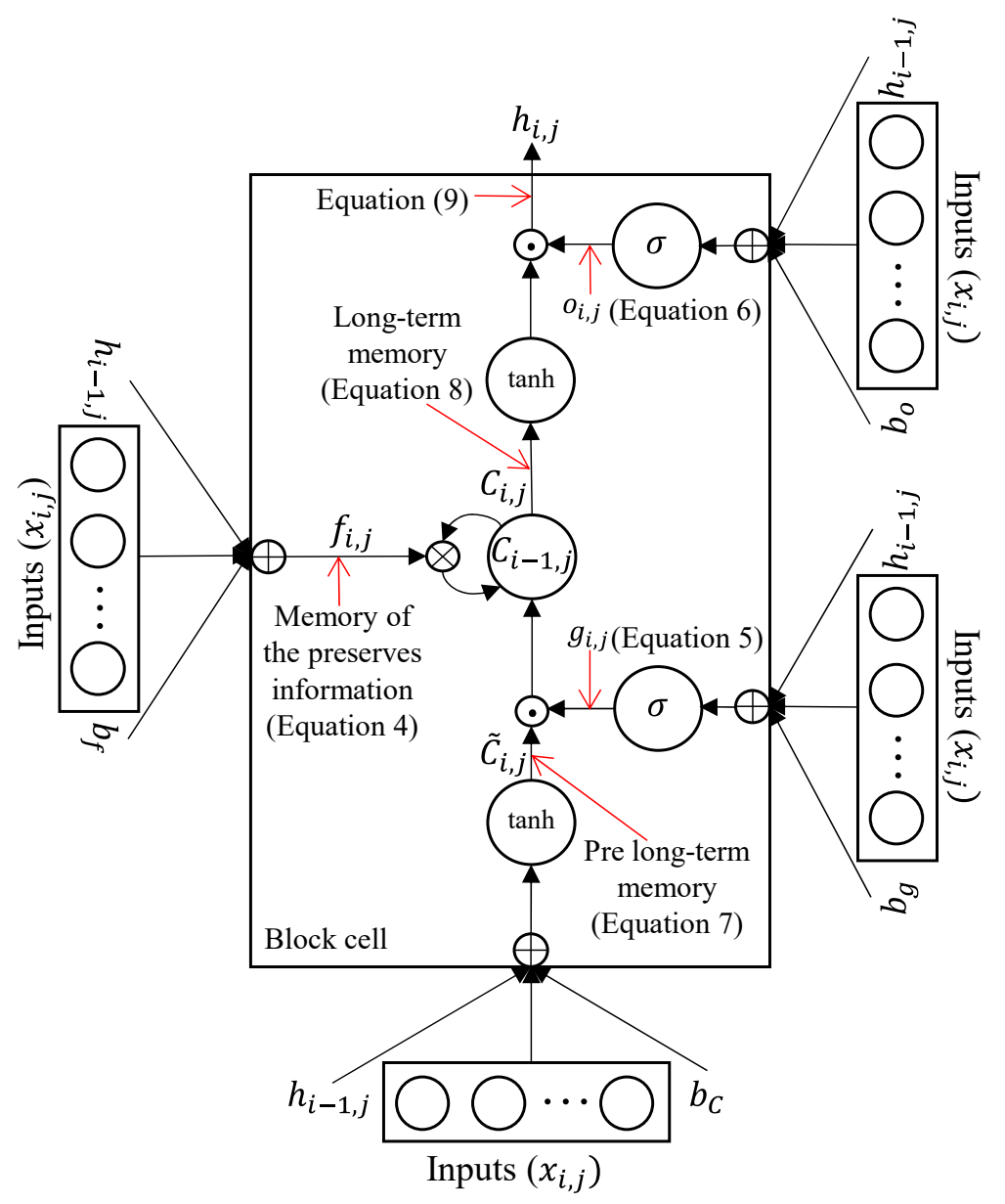

Figure 5. Block cell structure of the proposed LSTM model for hourly PV power prediction.

For calculating the output for a gate, the logistic sigmoid function is applied, denoted as $\sigma$, for the weighted summation results with respect to the current inputs, the past outputs, and the bias values for the gate. In summary, the gate outputs in LSTM block cell are calculated as Equations (4)-(6): 


$$
\begin{aligned}
& f_{i, j}=\sigma\left(W_{x_{i, j}} x_{i, j}+W_{h f} h_{i-1, j}+b_{f}\right) \\
& g_{i, j}=\sigma\left(W_{x_{i, j}} x_{i, j}+W_{h g} h_{i-1, j}+b_{g}\right) \\
& o_{i, j}=\sigma\left(W_{x_{i, j}} x_{i, j}+W_{h o} h_{i-1, j}+b_{o}\right)
\end{aligned}
$$

To obtain long term patterns for a specific hour that represent the long-term relationship among PV power outputs across days, the input and forget gates are involved. In particular, for the $i$-th hour in the $j$-th day, $c_{i, j}$, based on the input gate output, $f_{i, j}$, and the forget gate output, $g_{i, j}$, as defined in Equation (7), and the operation "." is a Hadamard multiplication:

$$
C_{i, j}=f_{i, j} \cdot C_{i-1, j}+\widetilde{C}_{i, j} \cdot g_{i, j}
$$

In Equation (7), $\widetilde{C}_{i, j}=\tanh \left(W_{x_{i, j} g} x_{i, j}+W_{h g} h_{i-1, j}+b_{c}\right)$ means the pre-long term information is calculated based on the current observation and the short term pattern for the previous hour. That is, $f_{i, j}$ and $g_{i, j}$ jointly determine the usage level of the current input information for the next long term pattern.

The final output of the block cell for the $i$-th hour of the $j$-th day, $h_{i, j}$, is simply examined based on the output gate value, $o_{i, j}$, and the long term pattern, $c_{i, j}$, presented in Equation (8):

$$
h_{i, j}=o_{i, j} \cdot \tanh \left(C_{i, j}\right)
$$

Figure 6 shows the entire structure of the proposed LSTM model in terms of the previously explained concept of the block cell. The input and output values used in the proposed LSTM model are zero-one normalized to resolve the inconsistencies among units [52]. To take advantage of multilayer LSTM that enables to capture the longer-term dependencies of input sequences [53], three block cells are used for each input and a block cell for a previous hour is connected to another block cell for the next hour. There are many learning algorithms for LSTM; we use the backpropagation through time method (BPTT) with the Adagrad optimizer [54-56]. The activation function and loss function used ReLU and MSE like the proposed ANN- and DNN-based models.

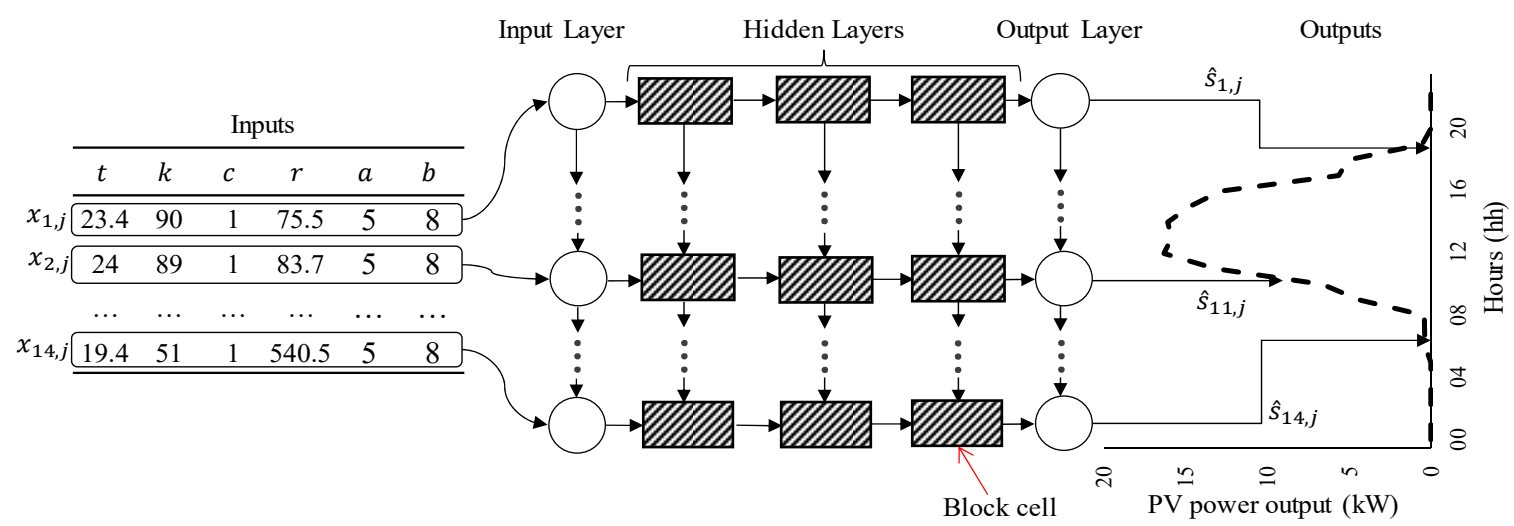

Figure 6. Overall structure of the proposed LSTM model for hourly PV power prediction.

\section{Experiments}

For the experiments of this research, we used the deep learning toolkit Keras. Among the famous deep learning toolkits such as Keras, Tensorflow, Theano, and Pytorch, Keras has a great benefit due to being easy and simple. Moreover, we used parameters such as optimizer, activation and loss function necessary of learning offered in the Keras library. Our computer resources consist of an i7-6700CPU, 8 GB RAM, and GPU1080. The learning and experiments phase of the proposed models were conducted in in the GPU environment. 
The dataset used for experiments was gathered from a PV power output dataset during 39 months (from 1 June 2013 to 31 August 2016) from a PV operator located in Gumi city in South Korea. Among the entire 16,820 instances in the dataset, 11,510 instances observed from 1 June 2013 to 31 August 2015 were used for training the considered prediction models, while the remainder were regarded as unseen observations and 3616 and 1694 used for validating and testing the performances.

Table 3 shows the examples of the dataset used in these experiments. The unit of temperature value is Celsius, and cloudiness values ranges from 0 to 10 which the higher number means the more clouds in the air. Further, the value of humidity ranges from 0 to 100 where the higher value means more humidity in the sky. These meteorological data related to the area where the PV operator is located were obtained from the open dataset provided by the Korea government [57].

Table 3. Obtained input and output values for learning proposed models (11 August 2015).

\begin{tabular}{|c|c|c|c|c|c|c|c|}
\hline \multicolumn{7}{|c|}{ Input Values } & \multirow{2}{*}{$\begin{array}{c}\text { Output Value } \\
\text { Actual PV Power } \\
\text { Output (kW) }\end{array}$} \\
\hline Hour & $\begin{array}{c}\text { Temperature } \\
\left({ }^{\circ} \mathrm{C}\right)\end{array}$ & $\begin{array}{l}\text { Humidity } \\
(\%)\end{array}$ & $\begin{array}{l}\text { Cloudiness } \\
\text { Index }\end{array}$ & $\begin{array}{l}\text { Radiation } \\
\left(\mathrm{W} / \mathrm{m}^{2}\right)\end{array}$ & $\begin{array}{l}\text { Month of } \\
\text { Year (-) }\end{array}$ & $\begin{array}{c}\text { Day of } \\
\text { Month (-) }\end{array}$ & \\
\hline 06 & 23.4 & 93 & 1 & 0 & $M$ & $\mathrm{D}$ & 0 \\
\hline 07 & 24.3 & 90 & 1 & 93.1 & M & $\mathrm{D}$ & 2 \\
\hline 08 & 26 & 85 & 1 & 249.7 & M & $\mathrm{D}$ & 5 \\
\hline 09 & 27.5 & 78 & 1 & 346.1 & M & $\mathrm{D}$ & 6 \\
\hline 10 & 29.7 & 68 & 1 & 408.9 & M & $\mathrm{D}$ & 9 \\
\hline 11 & 30.8 & 65 & 1 & 447.1 & M & $\mathrm{D}$ & 11 \\
\hline 12 & 33.6 & 52 & 2 & 677.7 & M & $\mathrm{D}$ & 18 \\
\hline 13 & 33 & 55 & 3 & 656.5 & M & $\mathrm{D}$ & 20 \\
\hline 14 & 33.1 & 53 & 3 & 663.9 & M & $\mathrm{D}$ & 18 \\
\hline 15 & 31.4 & 55 & 4 & 592.5 & M & $\mathrm{D}$ & 17 \\
\hline 16 & 32.1 & 53 & 3 & 423.5 & M & $\mathrm{D}$ & 11 \\
\hline 17 & 30.9 & 62 & 3 & 323.4 & M & $\mathrm{D}$ & 8 \\
\hline 18 & 29.3 & 63 & 3 & 196.3 & M & $\mathrm{D}$ & 3 \\
\hline 19 & 27.2 & 71 & 1 & 45.5 & M & $\mathrm{D}$ & 0 \\
\hline
\end{tabular}

In the training phase, as the performance of proposed models varies depending on batch size [58,59], the batch size for the proposed ANN- and DNN-based models was set to 128, and it was set to 1 for the proposed LSTM-based model. We set the number of training epochs for the ANNand DNN-based model to be 250 while it was 25 for the LSTM-based model. The initial values for the weights of all the proposed models were randomly set between zero and one. The performance of the proposed models depends on overfitting or underfitting, according to the number of epochs. Following the suggestions in $[60,61]$, through many experimental trials, we determined the above settings minimizing the over- and underfitting problems while maximizing the generalization ability of the proposed models in terms of the loss value obtained using Equation (3).

Figure 7 presents the changes of the loss values for the three proposed models according to epochs during the training phase. The decreased loss values of training and validation as the number of epochs increases shown in the three graphs indicate that our models successfully attempt to search for better solutions against the given training dataset.

In addition, for comparison purposes, we implemented two popular statistical time-series models, called ARIMA and S-ARIMA, and two deep learning-based models, presented as DNN2 and LSTM2 in this paper. DNN2 model consists of a single input for the meteorological and seasonal information of a particular hour and an output resulting in the PV power output for the hour. The LSTM2 model attempts to predict PV power output by only using meteorological information without seasonal information [26,41,62]. ARIMA and S-ARIMA are widely used in time-series analysis and forecasting applications. ARIMA attempts to predict the future values based on auto-regressive and moving average techniques $[63,64]$. Unlike ARIMA, that focuses only on a few number of past instances observed just before, S-ARIMA exploits a larger number of past instances to consider the seasonal 
patterns throughout them [65]. The parameters for the two models were set to be the same as presented in $[64,66]$.

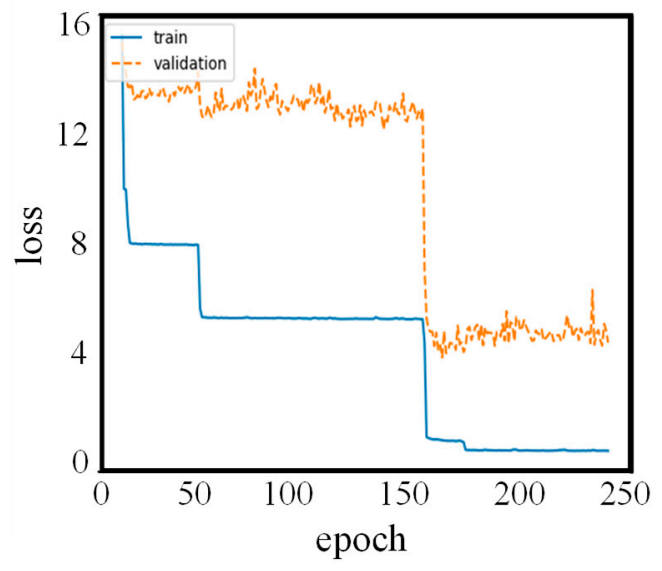

(a) ANN-based model

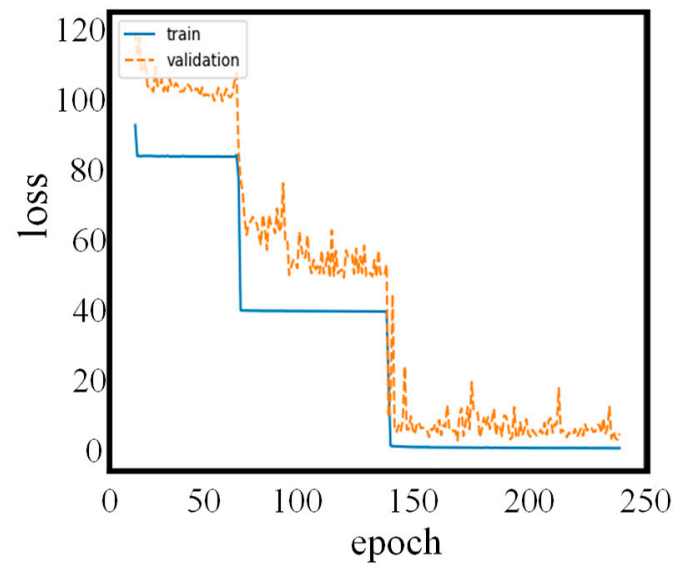

(b) DNN-based model

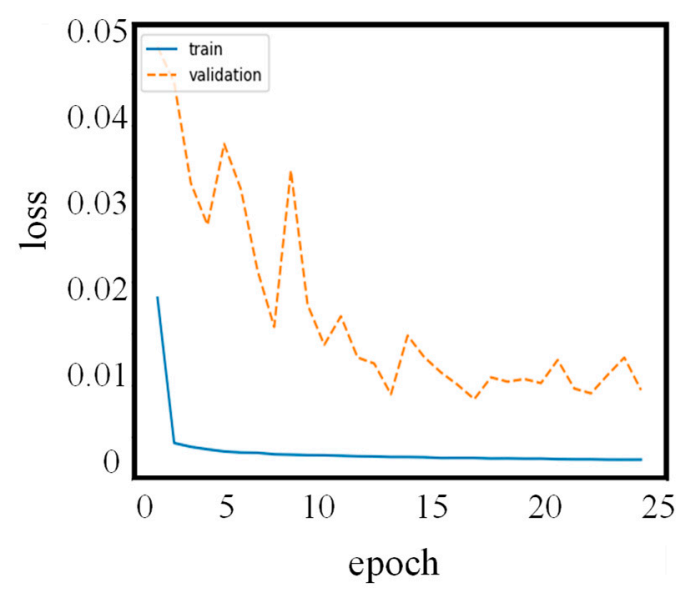

(c) LSTM-based model

Figure 7. Changes of the loss values according to the number of epochs during the training phase.

To quantify how well a prediction model estimate actual outputs, we calculated the mean absolute error $(M A E)$ based on the difference between each predicted PV power output for a given input instance against its actual value. Specifically, the mean absolute error, denoted as $M A E$, for the testing dataset is obtained by using Equation (9). Here, in the case of the actual PV power is zero, since the value becomes infinite, we set the error to be zero:

$$
M A E=\frac{1}{m^{\prime}} \sum_{i=1}^{m^{\prime}} \frac{\sum_{j=1}^{n}\left|s_{i, j}-\hat{s}_{i, j}\right|}{\sum_{j=1}^{n} s_{i, j}}
$$

where $m^{\prime}$ is the number of instances used in the testing phase.

Figure 8 compares the overall performances of the considered prediction models. The proposed ANN-based model is quite less effective compared to the conventional methods, ARIMA, S-ARIMA, DNN2, and LSTM2 models, while the proposed DNN-based model shows better performances compared to both the ANN-based model and the conventional ones. Overall, MAE was decreased $19 \%, 26 \%, 18 \%$ and $40 \%$ by the proposed DNN-based model compared to ARIMA, S-ARIMA, DNN2, and LSTM2, respectively. Such poor performance of the ANN-based model implies the limitation of the relationships between the current observation and its expected output without considering the time-series patterns. 


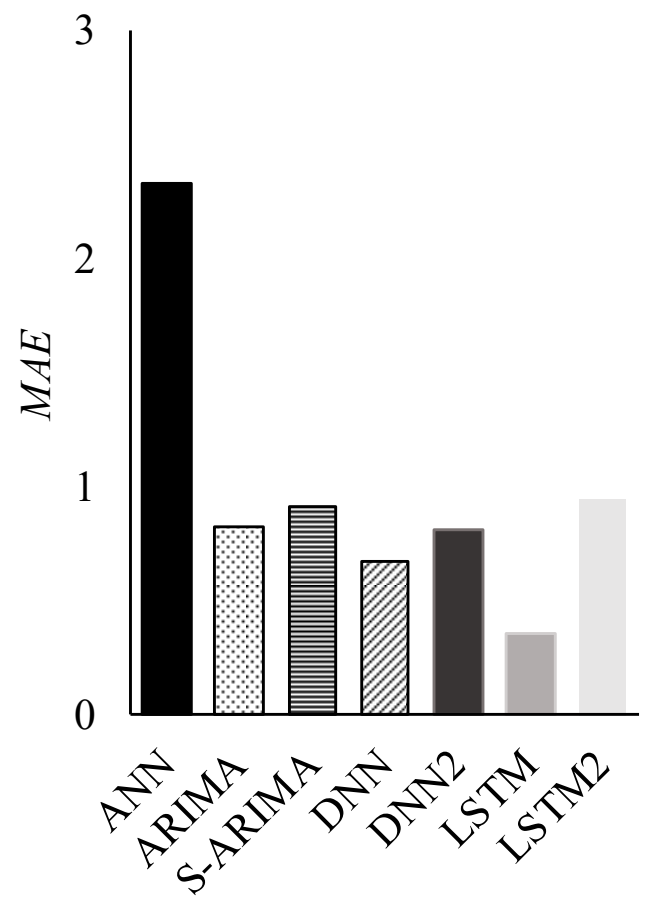

Figure 8. Performance comparisons using the testing dataset in terms of mean absolute error (MAE) (DNN and the LSTM are the proposed models).

Much better performances are obtained by the proposed LSTM-based model compared to all the models considered through exploiting both the long and short term patterns hidden in the past PV power outputs. The proposed LSTM-based model significantly reduces MAE by $80 \%$ compared to the ANN-based model, and $56 \%, 60 \%, 55 \%$, and $61 \%$ of the performances are improved compared to ARIMA, S-ARIMA, DNN2, and LSTM2, respectively. The improvements of LSTM-based model are $53 \%$ against the DNN-based model with respect to $M A E$.

In the short term perspective that focuses on the hourly changes of PV power outputs in a day, Figure 9 depicts how well the proposed methods produce robust performances. Here, to compare the prediction performances according to the difficulty levels of days, when (i) its PV power is increased compared to that of its previous PV power and decreased compared to its next PV power or (ii) its $\mathrm{PV}$ power is decreased compared to that of its previous $\mathrm{PV}$ power and increased compared to its next PV power. In this cases, the number of changes of PV power outputs for each day is calculated. It is reasonable since more changes of $\mathrm{PV}$ power output means much difficulty to predict. The experimental results show the outstanding performances of the proposed LSTM-based model compared to the others, especially when the prediction difficulty level becomes high.

As the difficulty level becomes higher, the errors of the prediction models tend to increase, especially when the number of PV power output changes is larger than four. The proposed ANNbased model shows the worst performances among the models considered in many cases, and the proposed DNN- and LSTM-based model yield quite low errors compared to the conventional models for all cases. For ARIMA, S-ARIMA, DNN, DNN2, and LSTM2 more than 1\% errors are frequently observed for the days with many PV power output changes, while the proposed LSTM-based model yields quite low errors even for these days. Especially, the proposed LSTM-based model shows successful results regardless of the number of PV power output changes and produces the quite impressive results for the days with the larger number of PV power output changes.

Such results imply that the architecture of LSTM that is designed to capture short and long term patterns is quite effective to accommodate not only the cases with few changes of PV power outputs but also the cases with many PV power output changes. On the contrary, the previous statistical prediction models, ARIMA and S-ARIMA, which are based on the direct dependency of consecutive 
observations, fail to precisely predict PV power outputs, especially for the cases with many PV power output changes.
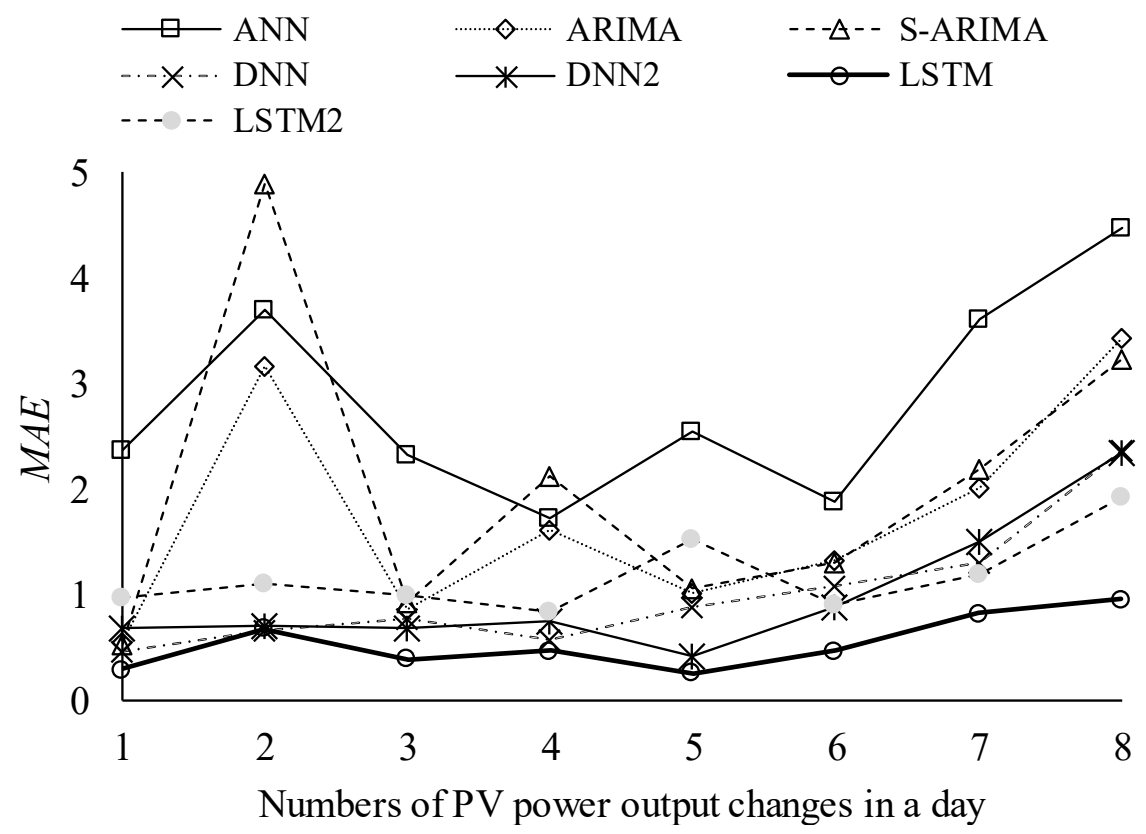

Figure 9. Distribution of mean absolute error $(M A E)$ according to the hourly changes of PV power output in a day.

Figure 10 visualizes the $M A E$ values of the predicted PV power outputs according to the numbers of hourly PV power output changes. As shown in Figure 10a, when the number of PV power output changes is low, quite successful results are observed for all the models considered except the proposed ANN-based model. However, when the number of PV power output changes becomes larger, as shown in Figure 10a,b, many more errors are obtained. When the number of PV power output changes is seven, as shown in Figure 10c, only the proposed LSTM-based model yields successful results. This means that the simple approach that considers only the relationships between meteorological information and PV power outputs shows limited performance when the prediction difficulty becomes high. On the other hand, overall, the proposed LSTM-based model results in the most accurate predictions.

In the long term perspective that concerns about changes of PV power outputs across days, we measured the difference between the amount of PV power outputs produced in a day and its consecutive day. For the four seasons, spring, summer, autumn, and winter, the average change of PV power outputs between days were calculated as $33.2 \%, 19.2 \%, 24.7 \%$, and $22.9 \%$. Therefore, as any two consecutive days in a season show larger changes in terms of the amount of the daily PV power output, the spring and autumn are considered as hard seasons compared to the other remaining seasons, considered as easy seasons.

Figure 11 illustrates the prediction performances of the models considered according to the easy and hard seasons. Here, plus means improved performance, while minus means worse performance. The higher values of the mean absolute error are observed in the easy season than in the hard seasons for all the models. On the average, $M A E$ is increased by $23.8 \%$ when predicting PV power outputs for the hard seasons compared to the easy seasons. 


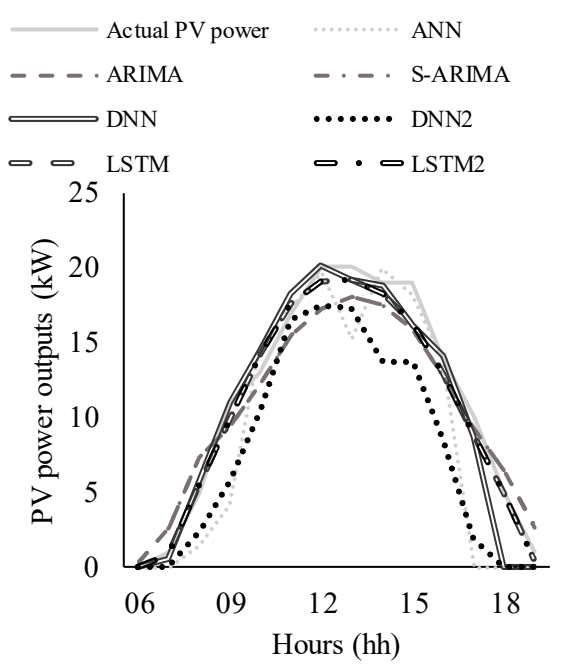

—ANN $\boxplus$ ARIMA $\boxminus$ S-ARIMA $\square$ DNN $\square$ DNN2 $\square$ LSTM $\square$ LSTM2

(a) When the number of PV power outputs changes is one (13 August 2016)
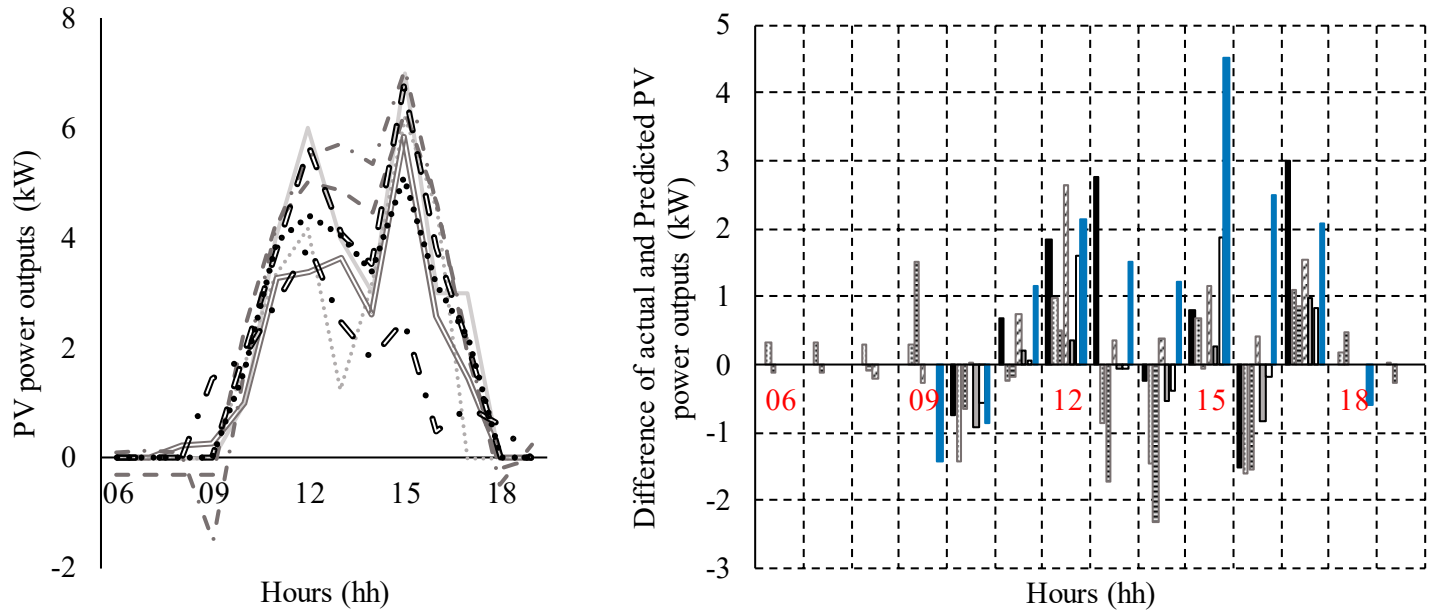

(b) When the number of PV power outputs changes is three (8 October 2016)
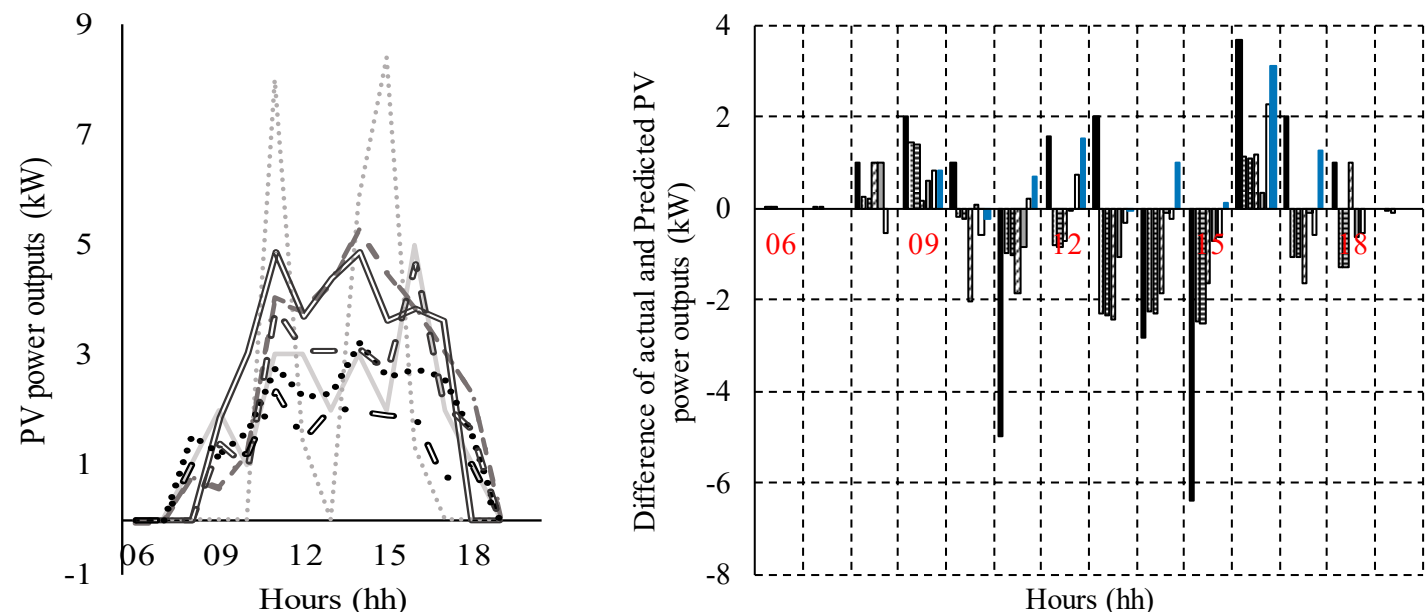

(c) When the number of PV power outputs changes is seven (4 April 2016)

Figure 10. Examples of predicted PV power results of according to the numbers of PV power output changes. 


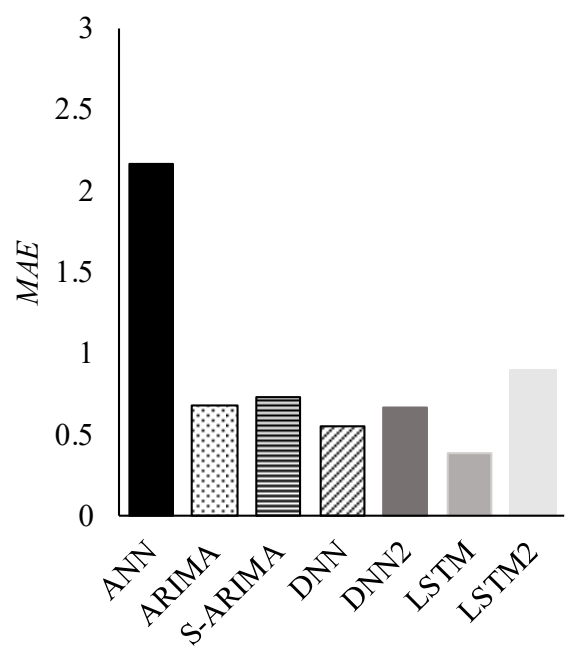

(a) Easy seasons

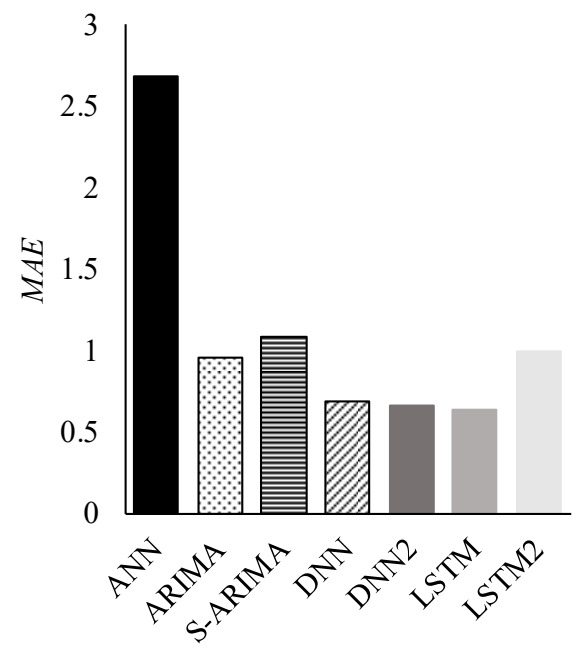

(b) Hard seasons

Figure 11. Performance comparisons according to easy and hard seasons.

Although the proposed LSTM-based model performs worse for the hard seasons compared to the easy seasons, its improvements compared to the others become larger as shown in Table 4 . The LSTM-based model outperforms the others by $60.4 \%$ on average in the easy seasons, while it shows $62 \%$ in the hard seasons in terms of MAE. Table 5 shows the detailed experimental results according to the seasons and the models considered in this paper.

Table 4. Improved ratio performance of between applied methods on easy and hard seasons.

\begin{tabular}{lcccccccc}
\hline & \multicolumn{7}{c}{ Improvement Ratios (\%) } \\
\cline { 3 - 8 } & & ANN & ARIMA & S-ARIMA & DNN & DNN2 & LSTM & LSTM2 \\
\hline \multirow{6}{*}{ Against } & ANN & - & +68.657 & +66.468 & +75.283 & +68.897 & +85.882 & +58.666 \\
& ARIMA & - & - & -6.984 & +21.138 & +1.374 & +54.957 & -23.075 \\
& S-ARIMA & - & - & - & +26.287 & +7.889 & +57.898 & -17.894 \\
& DNN & - & - & - & - & -22.843 & +42.883 & -38.746 \\
& DNN2 & - & - & - & - & - & +56.506 & -27.753 \\
& LSTM & - & - & - & - & - & - & -62.912 \\
& LSTM2 & - & - & - & - & - & - & - \\
\hline
\end{tabular}

(a) Performance improvements among the methods applied for the easy seasons

\begin{tabular}{lcccccccc}
\hline & \multicolumn{7}{c}{ Improvement Ratios (\%) } \\
\cline { 3 - 9 } & & ANN & ARIMA & S-ARIMA & DNN & DNN2 & LSTM & LSTM2 \\
\hline \multirow{6}{*}{ Against } & ANN & - & +60.905 & +56.147 & +67.765 & +73.693 & +74.017 & +63.058 \\
& ARIMA & - & - & -12.169 & +29.548 & +31.549 & +34.559 & -3.159 \\
& S-ARIMA & - & - & - & +33.493 & +35.357 & +37.272 & +8.717 \\
& DNN & - & - & - & - & +3.492 & +7.314 & -45.443 \\
& DNN2 & - & - & - & - & - & +4.061 & -50.706 \\
& LSTM & - & - & - & - & - & - & -57.086 \\
& LSTM2 & - & - & - & - & - & - & - \\
\hline
\end{tabular}

(b) Performance improvements among the methods applied for the hard seasons

Figure 12 visualizes the predicted PV power results for three pairs of consecutive two days. To demonstrate the ability to accommodate on the dynamic changes of PV power outputs across days, we choose examples based on the changes of the actual PV power outputs on consecutive days. First, when the amount of PV power output is similar between days, most of the considered models show quite successful results. On the other hand, as seen in Figure 10b,c, as the PV power output change for the second day is larger, the proposed LSTM-based model shows better performances compared to 
the others. That is, the efforts that consider the long term patterns between days rather than the short term patterns in a day are helpful for further precise predictions, especially when there are unstable $\mathrm{PV}$ power outputs among days.

Table 5. Details of the performances according to seasons in 2016 (underline means the highest performance for each week).

\begin{tabular}{|c|c|c|c|c|c|c|c|c|}
\hline \multirow{2}{*}{ Month of Year } & \multirow{2}{*}{ Week of Month } & \multicolumn{7}{|c|}{$M A E$} \\
\hline & & ANN & ARIMA & S-ARIMA & DNN & DNN2 & LSTM & LSTM2 \\
\hline \multirow{4}{*}{$\begin{array}{l}\text { Spring } \\
\text { (April) }\end{array}$} & 1 & 4.539 & 1.214 & 1.216 & 1.409 & 1.049 & $\underline{0.686}$ & 0.998 \\
\hline & 2 & 2.099 & 1.013 & 1.049 & 0.823 & 0.848 & $\overline{0.414}$ & 1.122 \\
\hline & 3 & 2.677 & 0.916 & 0.933 & 0.710 & 0.782 & $\overline{0.409}$ & 1.174 \\
\hline & 4 & 2.015 & 0.934 & 0.929 & 0.533 & 0.785 & $\underline{0.283}$ & 1.062 \\
\hline \multirow{4}{*}{$\begin{array}{l}\text { Summer } \\
\text { (August) }\end{array}$} & 1 & 2.451 & 0.390 & 0.384 & 0.511 & 0.791 & $\underline{0.318}$ & 0.864 \\
\hline & 2 & 2.328 & 0.425 & 0.407 & 0.486 & 0.878 & $\overline{0.330}$ & 0.894 \\
\hline & 3 & 2.132 & 0.353 & 0.335 & 0.493 & 0.845 & 0.239 & 0.799 \\
\hline & 4 & 2.610 & 1.131 & 1.300 & 0.468 & 0.605 & $\underline{0.263}$ & 0.924 \\
\hline \multirow{4}{*}{$\begin{array}{l}\text { Autumn } \\
\text { (October) }\end{array}$} & 1 & 2.359 & 0.655 & 0.815 & 0.753 & 0.747 & $\underline{0.468}$ & 1.035 \\
\hline & 2 & 2.040 & 0.592 & 0.582 & 0.736 & 0.683 & $\overline{0.296}$ & 0.955 \\
\hline & 3 & 2.200 & 0.484 & 0.447 & 0.957 & 0.489 & $\underline{0.244}$ & 1.185 \\
\hline & 4 & 1.997 & 1.982 & 2.767 & $\underline{0.502}$ & 0.596 & 0.584 & 0.924 \\
\hline \multirow{4}{*}{$\begin{array}{l}\text { Winter } \\
\text { (March) }\end{array}$} & 1 & 1.883 & 0.941 & 1.267 & 0.422 & 1.045 & 0.319 & 0.801 \\
\hline & 2 & 1.902 & 0.741 & 0.777 & 0.755 & 0.804 & 0.389 & 0.857 \\
\hline & 3 & 2.005 & 0.874 & 0.842 & 0.666 & 0.775 & $\underline{0.285}$ & 0.994 \\
\hline & 4 & 2.001 & 0.571 & 0.493 & 0.478 & 1.356 & $\underline{0.301}$ & 1.197 \\
\hline
\end{tabular}

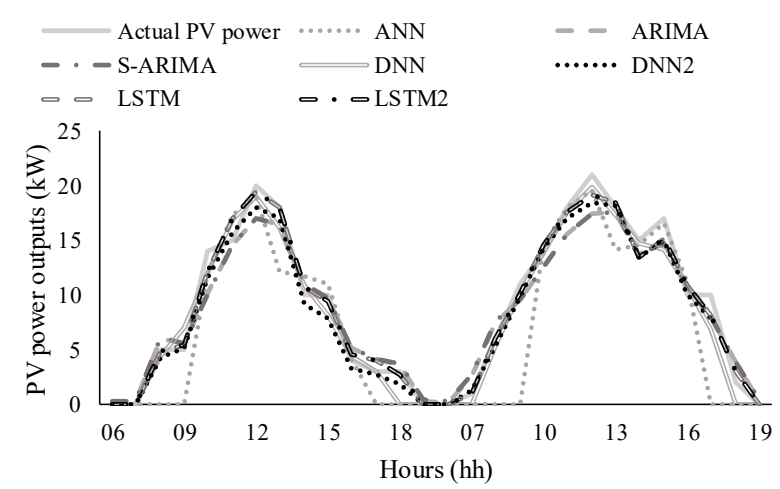

\begin{tabular}{|c|c|c|c|c|c|c|c|c|c|}
\hline \multirow{8}{*}{$\begin{array}{c}17 \\
\text { August } \\
2016\end{array}$} & Hours & $\begin{array}{c}\text { Actual PV } \\
\text { Power Output }\end{array}$ & ANN & ARIMA & S-ARIMA & DNN & DNN2 & LSTM & LSTM2 \\
\hline & $\ldots$ & $\ldots$ & $\ldots$ & $\ldots$ & $\ldots$ & $\ldots$ & $\ldots$ & $\ldots$ & $\ldots$ \\
\hline & $12: 00$ & 20 & 17.39 & 14.55 & 17.03 & 19.05 & 18.11 & 19.47 & 19.47 \\
\hline & 13:00 & 18 & 19.09 & 17.01 & 16.49 & 16.17 & 16.98 & 18.03 & 17.03 \\
\hline & $14: 00$ & 10 & 12.22 & 16.4 & 11.01 & 10.91 & 9.15 & 10.68 & 11.28 \\
\hline & $15: 00$ & 10 & 11.78 & 10.96 & 9.74 & 8.17 & 7.86 & 9.47 & 9.17 \\
\hline & $16: 00$ & 4 & 4.15 & 9.71 & 5.19 & 4.49 & 3.29 & 4.63 & 4.32 \\
\hline & $\ldots$ & $\ldots$ & $\ldots$ & $\ldots$ & $\ldots$ & $\ldots$ & $\ldots$ & $\ldots$ & $\ldots$ \\
\hline \multirow{7}{*}{$\begin{array}{c}18 \\
\text { August } \\
2016\end{array}$} & $\ldots$ & $\ldots$ & $\ldots$ & $\ldots$ & $\ldots$ & $\ldots$ & $\ldots$ & $\ldots$ & $\ldots$ \\
\hline & $12: 00$ & 21 & 19.86 & 19.57 & 19.20 & 17.45 & 18.49 & 18.46 & 19.20 \\
\hline & 13:00 & 18 & 17.28 & 14.22 & 18.50 & 17.56 & 18.13 & 17.57 & 18.50 \\
\hline & $14: 00$ & 15 & 14.78 & 14.61 & 13.51 & 13.68 & 13.43 & 13.70 & 13.51 \\
\hline & $15: 00$ & 17 & 14.13 & 16.64 & 15.12 & 14.77 & 15.02 & 14.79 & 15.12 \\
\hline & $16: 00$ & 10 & 10.78 & 11.21 & 10.85 & 10.73 & 9.97 & 10.74 & 10.85 \\
\hline & $\ldots$ & $\ldots$ & $\ldots$ & $\ldots$ & $\ldots$ & $\ldots$ & $\ldots$ & $\ldots$ & $\ldots$ \\
\hline
\end{tabular}

(a) When the PV power outputs of two consecutive days are similar (17, 18 August 2016)

Figure 12. Cont. 


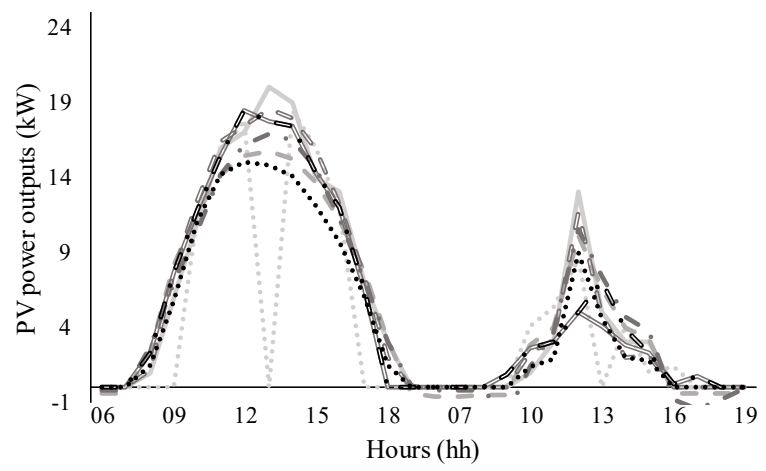

\begin{tabular}{|c|c|c|c|c|c|c|c|c|c|}
\hline \multirow{8}{*}{$\begin{array}{c}17 \\
\text { October } \\
2016\end{array}$} & Hours & $\begin{array}{c}\text { Actual PV } \\
\text { power } \\
\text { output }\end{array}$ & ANN & ARIMA & S-ARIMA & DNN & DNN2 & LSTM & LSTM2 \\
\hline & $\ldots$ & $\ldots$ & $\ldots$ & $\ldots$ & $\ldots$ & $\ldots$ & $\ldots$ & $\ldots$ & $\ldots$ \\
\hline & $12: 00$ & 17 & 17.79 & 15.49 & 16.15 & 18.47 & 15.03 & 17.46 & 18.47 \\
\hline & 13:00 & 20 & 0.00 & 15.71 & 16.85 & 17.78 & 14.83 & 18.59 & 17.78 \\
\hline & $14: 00$ & 19 & 18.44 & 15.25 & 16.49 & 17.37 & 14.04 & 17.94 & 17.37 \\
\hline & 15:00 & 14 & 15.99 & 13.63 & 14.62 & 14.30 & 12.15 & 15.86 & 14.30 \\
\hline & $16: 00$ & 13 & 11.95 & 11.16 & 11.30 & 11.98 & 9.69 & 12.23 & 11.98 \\
\hline & $\ldots$ & $\ldots$ & $\ldots$ & $\ldots$ & $\ldots$ & $\ldots$ & $\ldots$ & $\ldots$ & $\ldots$ \\
\hline \multirow{7}{*}{$\begin{array}{c}18 \\
\text { October } \\
2016\end{array}$} & $\ldots$ & $\ldots$ & $\ldots$ & $\ldots$ & $\ldots$ & $\ldots$ & $\ldots$ & $\ldots$ & $\ldots$ \\
\hline & $12: 00$ & 13 & 8.58 & 10.12 & 10.56 & 5.05 & 8.97 & 11.72 & 7.07 \\
\hline & 13:00 & 5 & 0.00 & 6.63 & 7.38 & 4.07 & 4.66 & 4.83 & 3.83 \\
\hline & $14: 00$ & 3 & 4.07 & 3.98 & 4.74 & 2.84 & 1.99 & 2.04 & 2.28 \\
\hline & 15:00 & 3 & 1.03 & 3.27 & 3.77 & 2.28 & 1.76 & 2.07 & 0.24 \\
\hline & $16: 00$ & 0 & 1.44 & -0.41 & -0.79 & 0.24 & 9 & 0.00 & 0.73 \\
\hline & $\ldots$ & $\ldots$ & $\ldots$ & $\ldots$ & $\ldots$ & $\ldots$ & $\ldots$ & $\ldots$ & $\ldots$ \\
\hline
\end{tabular}

(b) When the PV power outputs of two consecutive days are largely different (17, 18 October 2016)

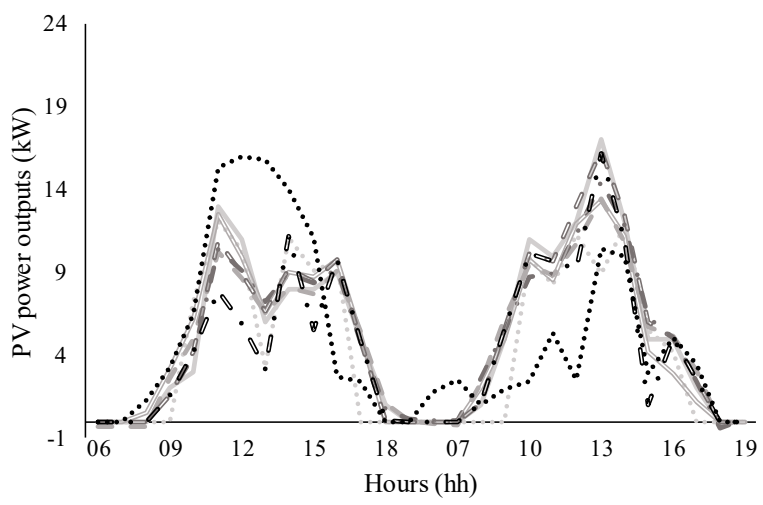

\begin{tabular}{|c|c|c|c|c|c|c|c|c|c|}
\hline \multirow{8}{*}{$\begin{array}{l}3 \text { March } \\
2016\end{array}$} & Hours & $\begin{array}{c}\text { Actual PV } \\
\text { power } \\
\text { output }\end{array}$ & ANN & ARIMA & S-ARIMA & DNN & DNN2 & LSTM & LSTM2 \\
\hline & $\ldots$ & $\ldots$ & $\ldots$ & $\ldots$ & $\ldots$ & $\ldots$ & $\ldots$ & $\ldots$ & $\ldots$ \\
\hline & $12: 00$ & 11 & 10.30 & 8.62 & 9.15 & 9.96 & 15.96 & 8.92 & 5.92 \\
\hline & 13:00 & 6 & 3.28 & 6.53 & 7.25 & 6.59 & 15.82 & 6.63 & 3.21 \\
\hline & $14: 00$ & 8 & 11.10 & 8.09 & 8.95 & 9.00 & 14.04 & 9.22 & 11.22 \\
\hline & $15: 00$ & 8 & 9.08 & 7.68 & 8.32 & 8.73 & 11.12 & 8.48 & 5.48 \\
\hline & $16: 00$ & 9 & 9.72 & 9.08 & 9.17 & 9.72 & 2.68 & 9.76 & 9.76 \\
\hline & $\ldots$ & $\ldots$ & $\ldots$ & $\ldots$ & $\ldots$ & $\ldots$ & $\ldots$ & $\ldots$ & $\ldots$ \\
\hline \multirow{7}{*}{$\begin{array}{l}4 \text { March } \\
2016\end{array}$} & $\ldots$ & $\ldots$ & $\ldots$ & $\ldots$ & $\ldots$ & $\ldots$ & $\ldots$ & $\ldots$ & $\ldots$ \\
\hline & $12: 00$ & 12 & 11.32 & 10.68 & 11.30 & 11.94 & 2.46 & 12.97 & 9.16 \\
\hline & $13: 00$ & 17 & 8.89 & 13.43 & 14.52 & 13.37 & 8.44 & 16.34 & 16.21 \\
\hline & 14:00 & 12 & 11.88 & 10.80 & 11.83 & 11.20 & 6.99 & 12.41 & 10.88 \\
\hline & $15: 00$ & 5 & 5.50 & 5.72 & 6.30 & 4.24 & 2.91 & 6.03 & 1.02 \\
\hline & $16: 00$ & 5 & 4.22 & 5.12 & 5.03 & 2.86 & 5.09 & 5.01 & 5.01 \\
\hline & $\ldots$ & $\ldots$ & $\ldots$ & $\ldots$ & $\ldots$ & $\ldots$ & $\ldots$ & $\ldots$ & $\ldots$ \\
\hline
\end{tabular}

(c) When the PV power outputs of two consecutive days are slightly different (3, 4 March 2016)

Figure 12. Visualizations of the predicted PV power outputs for consecutive two days. 
RMSE may more useful when the large errors measured are particularly undesirable [67]. For the performance evaluation of the proposed models, we additionally adopt to attempt the perform in terms of root mean square error (RMSE). Specifically, RMSE is calculated by using Equation (10):

$$
R M S E=\frac{1}{m^{\prime}} \sum_{i=1}^{m^{\prime}} \frac{\sum_{j=1}^{n} \sqrt{\left(s_{i, j}-\hat{s}_{i, j}\right)^{2}}}{\sum_{j=1}^{n} s_{i, j}}
$$

where $m^{\prime}$ is the number of instances used in the testing phase.

Table 6 compares the performances of the proposed models in terms of RMSE according to seasons. All the proposed models yield similar errors such as $M A E$, and outperform in summer like in previous research results [12]. The proposed ANN-based model performs in winter compared to the remaining seasons, while the proposed LSTM-based model yields quite fewer errors for all seasons.

Table 6. Performance comparisons in terms of RMSE according to seasons in 2016.

\begin{tabular}{cccccccc}
\hline \multicolumn{7}{c}{ RMSE } \\
\hline & ANN & ARIMA & S-ARIMA & DNN & DNN2 & LSTM & LSTM2 \\
\hline Spring (April) & 3.617 & 1.254 & 1.256 & 1.023 & 1.258 & 0.780 & 1.797 \\
\hline Summer (August) & 1.669 & 0.889 & 0.843 & 0.701 & 0.995 & 0.563 & 1.262 \\
\hline Autumn (October) & 3.118 & 1.251 & 1.240 & 1.044 & 1.061 & 0.698 & 1.677 \\
\hline Winter (March) & 8.015 & 1.829 & 1.450 & 1.096 & 1.592 & 0.874 & 1.816 \\
\hline
\end{tabular}

\section{Conclusions}

In this study, we have proposed a PV power prediction method based on neural networks. First, we suggest a simple ANN-based model that focuses only on the relationships between metrological information and PV power output for a particular hour. Next, a developed version of the firstly proposed model, a DNN-based model, is suggested by using more hidden layers. Finally, a more sophisticated model, a LSTM-based model, which considers both short and long term patterns in a day and across days, respectively, is proposed.

By using the experiment results from a real-world dataset, although the proposed ANN-based model fails to result in better performances compared to the conventional models, the proposed DNNand LSTM-based models outperform them. Especially, the LSTM-based model shows quite successful performances for most of the experiment settings. Moreover, for the days that involve many changes of PV power outputs, the proposed LSTM-based model yields much better results than the others.

In this paper, the PV power outputs on a particular day are assumed to be independent of those on another day, while the output at a particular hour depends on that during its previous hour. We note that incorporating information from previous hours and previous days would be helpful to improve prediction quality as suggested in [68], but it becomes very difficult due to the increased parameters to be learned. Therefore, to improve the performances of the proposed LSTM-based model in Section 2.3, we plan to utilize these information through enhancing techniques for more sophisticated prediction learns as a future study.

Further, based on the models suggested in this paper, we plan to further improve the proposed models towards PV power output prediction without metrological information. Since there still exist many areas where such detailed metrological information is unavailable, we believe that these prediction models are much practical.

Author Contributions: D.L. and K.K. conducted the initial design and developed the framework. D.L. wrote the draft of the manuscript, while K.K. reviewed and proof read the paper.

Funding: This work was supported by the Incheon National University (International Cooperative) Research Grant in 2015. 
Conflicts of Interest: The authors declare no conflict of interest.

\section{References}

1. Saw, L.H.; Poon, H.M.; Thiam, H.S.; Cai, Z.; Chong, W.T.; Pambudi, N.A.; King, Y.J. Novel Thermal Management System Using Mist Cooling for Lithium-ion Battery Packs. Appl. Energy 2018, 223, $146-158$. [CrossRef]

2. Ming, B.; Liu, P.; Cheng, L.; Zhou, Y.; Wang, X. Optimal Daily Generation Ccheduling of Large Yydro-Photovoltaic Hybrid Power Plants. Energy Convers. Manag. 2018, 171, 528-540. [CrossRef]

3. Deshmukh, M.K.; Deshmukh, S.S. Modeling of Hybrid Renewable Energy Systems. Renew. Sustain. Energy Rev. 2008, 12, 235-249. [CrossRef]

4. Paska, J.; Biczel, P.; Kłos, M. Hybrid Power Systems-An Effective Way of Utilising Primary Energy Sources. Renew. Energy 2009, 34, 2414-2421. [CrossRef]

5. Hoffmann, W. PV Solar Electricity Industry: Market Growth and Perspective. Sol. Energy Mater. Sol. Cells 2006, 90, 3285-3311. [CrossRef]

6. Pierro, M.; Bucci, F.; De Felice, M.; Maggioni, E.; Moser, D.; Perotto, A.; Spada, F.; Cornaro, C. Multi-Model Ensemble for Day Ahead Prediction of Photovoltaic Power Generation. Sol. Energy 2016, 134, $132-146$. [CrossRef]

7. Zhou, Y.; Wang, C.; Wu, J.; Wang, J.; Cheng, M.; Li, G. Optimal Scheduling of Aggregated Thermostatically Controlled Loads with Renewable Generation in the Intraday Electricity Market. Appl. Energy 2017, 188, 456-465. [CrossRef]

8. Ayón, X.; Moreno, M.Á.; Usaola, J. Aggregators' Optimal Bidding Strategy in Sequential Day-Ahead and Intraday Electricity Spot Markets. Energies 2017, 10, 450. [CrossRef]

9. Rowlands, I.H. Solar PV Electricity and Market Characteristics: Two Canadian Case-studies. Renew. Energy 2005, 30, 815-834. [CrossRef]

10. Yona, A.; Senjyu, T.; Funabashi, T.; Mandal, P.; Kim, C.-H. Optimizing Re-planning Operation for Smart House Applying Solar Radiation Forecasting. Appl. Sci. 2014, 4, 366-379. [CrossRef]

11. Inman, R.H.; Pedro, H.T.C.; Coimbra, C.F.M. Solar Forecasting Methods for Renewable Energy Integration. Prog. Energy Combust. Sci. 2013, 39, 535-576. [CrossRef]

12. Kardakos, E.G.; Alexiadis, M.C.; Vagropoulos, S.I.; Simoglou, C.K.; Biskas, P.N.; Bakirtzis, A.G. Application of Time Series and Artificial Neural Network Models in Short-term Forecasting of PV Power Generation. In Proceedings of the 48th International Universities Power Engineering Conference, Dublin, Ireland, 2-5 September 2013; pp. 1-6.

13. Cai, T.; Duan, S.; Chen, C. Forecasting Power Output for Grid-connected Photovoltaic Power System Without Using Solar Radiation Measurement. In Proceedings of the International Symposium on Power Electronics for Distributed Generation Systems, Hefei, China, 16-18 June 2010; pp. 773-777.

14. Yona, A.; Senjyu, T.; Funabashi, T.; Kim, C.H. Determination Method of Insolation Prediction with Fuzzy and Applying Neural Network for Long-term Ahead PV Power Output Correction. IEEE Trans. Sustain. Energy 2013, 2, 527-533. [CrossRef]

15. Dumitru, C.-D.; Gligor, A.; Enachescu, C. Solar Photovoltaic Energy Production Forecast Using Neural Networks. Procedia Technol. 2016, 22, 808-815. [CrossRef]

16. Izgi, E.; Öztopal, A.; Yerli, B.; Kaymak, M.K.; Şahin, A.D. Short-mid-term Solar Power Prediction by Using Artificial Neural Networks. Sol. Energy 2012, 86, 725-733. [CrossRef]

17. Sulaiman, S.I.; Rahman, T.K.A.; Musirin, I. Partial Evolutionary ANN for Output Prediction of a Grid-Connected Photovoltaic System. Int. J. Comput. Electr. Eng. 2009, 1, 40-45. [CrossRef]

18. Wang, S.; Zhang, N.; Zhao, Y.; Zhan, J. Photovoltaic System Power Forecasting Based on Combined Grey Model and BP Neural Network. In Proceedings of the International Conference on Electrical and Control Engineering, Yichang, China, 16-18 September 2011; pp. 4623-4626.

19. Son, J.; Park, Y.; Lee, J.; Kim, H. Sensorless PV Power Forecasting in Grid-Connected Buildings through Deep Learning. Sensors 2018, 18, 2529. [CrossRef] [PubMed]

20. Ashraf, I. Chandra, a Artificial Neural Ntwork Bsed Mdels for Frecasting Eectricity Gneration of Gid Connected Solar PV Power Plant. Int. J. Glob. Energy Issues 2004, 21, 119-130. [CrossRef] 
21. Shi, J.; Lee, W.J.; Liu, Y.; Yang, Y.; Wang, P. Forecasting Power Output of Photovoltaic Systems Based on Weather Classification and Support Vector Machines. IEEE Trans. Ind. Appl. 2012, 48, 1064-1069. [CrossRef]

22. Da Silva Fonseca, J.G.; Oozeki, T.; Takashima, T.; Koshimizu, G.; Uchida, Y.; Ogimoto, K. Use of Support Vector Regression and Numerically Predicted Cloudiness to Forecast Power Output of a Photovoltaic Power Plant in Kitakyushu, Japan. Proc. Photovolt. Res. Appl. 2012, 20, 874-882. [CrossRef]

23. Bouzerdoum, M.; Mellit, A.; Massi Pavan, A. A Hybrid Model (SARIMA-SVM) for Short-term Power Forecasting of a Small-scale Grid-connected Photovoltaic Plant. Sol. Energy 2013, 98, 226-235. [CrossRef]

24. Mellit, A.; Shaari, S. Recurrent Neural Network-Based Forecasting of The Daily Electricity Generation of a Photovoltaic Power System. In Proceedings of the Ecological Vehicle and Renewable Energy, Monte-Carlo, Monaco, 26-29 March 2009; pp. 26-29.

25. Mandal, P.; Madhira, S.T.S.; Ul haque, A.; Meng, J.; Pineda, R.L. Forecasting Power Output of Solar Photovoltaic System Using Wavelet Transform and Artificial Intelligence Techniques. Procedia Comput. Sci. 2012, 12, 332-337. [CrossRef]

26. Abdel-Nasser, M.; Mahmoud, K. Accurate Photovoltaic Power Forecasting Models Using Deep LSTM-RNN. In Neural Computing and Applications; Springer: London, UK, 2017; pp. 1-14.

27. Chattopadhyay, K.; Kies, A.; Lorenz, E.; von Bremen, L.; Heinemann, D. The Impact of Different PV Module Configurations on Storage and Additional Balancing Needs for a fully Renewable European Power System. Renew. Energy 2017, 113, 176-189. [CrossRef]

28. Jurasz, J.; Ciapała, B. Solar-hydro Hybrid Power Station as a Way to Smooth Power Output and Increase Water Retention. Sol. Energy 2018, 173, 675-690. [CrossRef]

29. Ding, M.; Wang, L.; Bi, R. An ANN-based Approach for Forecasting The Power Output of Photovoltaic System. Procedia Environ. Sci. 2011, 11, 1308-1315. [CrossRef]

30. Long, H.; Zhang, Z.; Su, Y. Analysis of Daily Solar Power Prediction with Data-driven Approaches. Appl. Energy 2014, 126, 29-37. [CrossRef]

31. Jiahao, K.; Jun, L.; Qifan, L.; Wanliang, F.; Zhenhuan, C.; Linlin, L.; Tieying, G. Photovoltaic Power Forecasting Based on Artificial Neural Network and Meteorological Data. In Proceedings of the IEEE Region 10 Conference, Xi'an, China, 22-25 October 2013; pp. 1-4.

32. Hiyama, T.; Kitabayashi, K. Neural Network Based Estimation of Maximum Power Generation from PV Module Using Environmental Information. IEEE Trans. Energy Convers. 1997, 12, 241-247. [CrossRef]

33. Wang, G.; Su, Y.; Shu, L. One-day-ahead Daily Power Forecasting of Photovoltaic Systems Based on Partial Functional Linear Regression Models. Renew. Energy 2016, 96, 469-478. [CrossRef]

34. Li, Y.; He, Y.; Su, Y.; Shu, L. Forecasting The Daily Power Output of a Grid-connected Photovoltaic System Based on Multivariate Adaptive Regression Splines. Appl. Energy 2016, 180, 392-401. [CrossRef]

35. Li, Y.; Su, Y.; Shu, L. An ARMAX Model for Forecasting The Power Output of a Grid Connected Photovoltaic System. Renew. Energy 2014, 66, 78-89. [CrossRef]

36. Mora-López, L.; Martinez-Marchena, I.; Piliougine, M.; Sidrach-deCardona, M. Machine Learning Approach for Next Day Energy Production Forecasting in Grid Connected Photovoltaic Plants. In Proceedings of the World Renewable Energy Congress-Sweden, Linköping, Sweden, 8-13 May 2011; pp. 2869-2874.

37. Al-Messabi, N.; Li, Y.; El-Amin, I.; Goh, C. Forecasting of Photovoltaic Power Yield Using Dynamic Neural Networks. In Proceedings of the International Joint Conference on Neural Networks, Brisbane, Australia, 10-15 June 2012; pp. 1-5.

38. Chen, C.; Duan, S.; Cai, T.; Liu, B. Online 24-h Solar Power Forecasting Based on Weather Type Classification Using Artificial Neural Network. Sol. Energy 2011, 85, 2856-2870. [CrossRef]

39. Yona, A.; Senjyu, T.; Saber, A.Y.; Funabashi, T.; Sekine, H.; Kim, C.H. Application of Neural Network to 24-hour-ahead Generating Power Forecasting for PV System. In Proceedings of the IEEE Power and Energy Society General Meeting: Conversion and Delivery of Electrical Energy in the 21st Century, Niigata, Japan, 5-8 November 2008; pp. 1-6.

40. Bengio, Y.; Simard, P.; Frasconi, P. Learning Long Term Dependencies with Gradient Descent is Difficult. IEEE Trans. Neural Netw. 1994, 5, 157-166. [CrossRef] [PubMed]

41. Gensler, A.; Henze, J.; Sick, B.; Raabe, N. Deep Learning for Solar Power Forecasting-An Approach Using AutoEncoder and LSTM Neural Networks. In Proceedings of the IEEE International Conference on Systems, Man, and Cybernetics, Budapest, Hungary, 9-12 October 2016; pp. 002858-002865. 
42. Wang, Y.; Shen, Y.; Mao, S.; Chen, X.; Zou, H. LASSO \& LSTM Integrated Temporal Model for Short-term Solar Intensity Forecasting. IEEE Internet Things J. 2018. [CrossRef]

43. Wang, F.; Yu, Y.; Zhang, Z.; Li, J.; Zhen, Z.; Li, K. Wavelet Decomposition and Convolutional LSTM Networks Based Improved Deep Learning Model for Solar Irradiance Forecasting. Appl. Sci. 2018, 8, 1286. [CrossRef]

44. Sorkun, M.C.; Paoli, C.; Incel, Ö.D. Time Series Forecasting on Solar Irradiation Using Deep Learning. In Proceedings of the 10th International Conference on Electrical and Electronics Engineering, Bursa, Turkey, 30 November-2 December 2017; pp. 151-155.

45. Wheather Data Release Portal. Available online: https://data.kma.go.kr/cmmn/main.do (accessed on 21 November 2018).

46. Garro, B.A.; Rodríguez, K.; Vázquez, R.A. Classification of DNA Microarrays Using Artificial Neural Networks and ABC Algorithm. Appl. Soft Comput. 2015. [CrossRef]

47. Pastur-Romay, L.A.; Cedrón, F.; Pazos, A.; Porto-Pazos, A.B. Deep Artificial Neural Networks and Neuromorphic Chips for Big Data Analysis: Pharmaceutical and Bioinformatics Applications. Int. J. Mol. Sci. 2016, 17, 1313. [CrossRef]

48. Krizhevsky, A.; Sutskever, I.; Hinton, G. Imagenet Classification with Deep Convolutional Neural Networks. Adv. Neural Inf. 2012, 25, 1-9. [CrossRef]

49. Hagan, M.T.; Menhaj, M.B. Training Feedforward Networks with The Marquardt Algorithm. IEEE Trans. Neural Netw. 1994, 5, 989-993. [CrossRef]

50. Kingma, D.P.; Ba, J.L. Adam: A Method for Stochastic Optimization. arXiv, 2014; arXiv:1412.6980.

51. Greff, K.; Srivastava, R.K.; Koutnik, J.; Steunebrink, B.R.; Schmidhuber, J. LSTM: A Search Space Odyssey. IEEE Trans. Neural Netw. Learn. Syst. 2017, 28, 2222-2232. [CrossRef]

52. Sola, J.; Sevilla, J. Importance of Input Data Normalization for the Application of Neural Networks to Complex Industrial Problems. IEEE Trans. Nucl. Sci. 1997, 44, 1464-1468. [CrossRef]

53. Zhang, S.; Liu, X.; Xiao, J. On Geometric Features for Skeleton-Based Action Recognition Using Multilayer LSTM Networks. In Proceedings of the IEEE Winter Conference on Applications of Computer Vision, Santa Rosa, CA, USA, 24-31 March 2017.

54. Williams, R.J.; Zipser, D. Gradient-Based Learning Algorithms for Recurrent Networks and Their Computational Complexity. BackPropag. Theory Archit. Appl. 1995, 1, 433-486. [CrossRef]

55. Graves, A. Generating Sequences with Recurrent Neural Networks. arXiv, 2013; arXiv:1308.0850. [CrossRef]

56. Duchi, J.; Hazan, E.; Singer, Y. Adaptive Subgradient Methods for Online Learning and Stochastic Optimization. J. Mach. Learn. Res. 2011, 12, 2121-2159. [CrossRef]

57. Astronomy Space Information of Korea Astronomy and Space Science Institute. Available online: https: / / astro.kasi.re.kr/life/pageView / 6 (accessed on 21 November 2018).

58. Dean, J.; Corrado, G.S.; Monga, R.; Chen, K.; Devin, M.; Le, Q.V.; Mao, M.Z.; Ranzato, M.A.; Senior, A.; Tucker, P.; et al. Large Scale Distributed Deep Networks. Adv. Neural Inf. Process. Syst. 2012. [CrossRef]

59. Masters, D.; Luschi, C. Revisiting Small Batch Training for Deep Neural Networks. arXiv 2018, arXiv:1804.07612v1.

60. Li, M.; Zhang, T.; Chen, Y.; Smola, A.J. Efficient Mini-batch Training for Stochastic Optimization. In Proceedings of the 20th ACM SIGKDD International Conference on Knowledge Discovery and Data Mining, New York, NY, USA, 24-27 August 2014; pp. 661-670.

61. Glorot, X.; Bengio, Y. Understanding the Difficulty of Training Deep Feedforward Neural Networks. In Proceedings of the Thirteenth International Conference on Artificial Intelligence and Statistics, Sardinia, Italy, 13-15 May 2010; Volume 9, pp. 249-256.

62. Cui, S. Solar Energy prediction and task scheduling for wireless sensor nodes based on long short term memory. J. Phys. 2018, 1074, 1-14. [CrossRef]

63. Zhang, G.P. Time Series Forecasting Using a Hybrid ARIMA and Neural Network Model. Neurocomputing 2003, 50, 159-175. [CrossRef]

64. Box, G.E.P.; Jenkins, G.M.; Reinsel, G.C.; Ljung, G.M. Time Series Analysis: Forecasting E Control; Wiley: New York, NY, USA, 2015; ISBN 8131716333.

65. Yu, L.; Zhou, L.; Tan, L.; Jiang, H.; Wang, Y.; Wei, S.; Nie, S. Application of a New Hybrid Model with Seasonal Auto-regressive Integrated Moving Average (ARIMA) and Nonlinear Auto-regressive Neural Network (NARNN) in Forecasting Incidence Cases of HFMD in Shenzhen, China. PLoS ONE 2014, 9, e98241. [CrossRef] [PubMed] 
66. Yule, G.U. Why do we Sometimes get Nonsense-Correlations between Time-Series?-A Study in Sampling and the Nature of Time-Series. J. R. Stat. Soc. 1926, 89, 1-63. [CrossRef]

67. Willmott, C.J.; Matsuura, K. Advantages of the Mean Absolute Error (MAE) Over the Root Mean Square Error (RMSE) in Assessing Average Model Performance. Clim. Res. 2005, 30, 79-82. [CrossRef]

68. Zhang, Y.; Wang, J. GEFCom2014 Probabilistic Solar Power Forecasting Based on k-nearest Neighbor and Kernel Density Estimator. In Proceedings of the Power \& Energy Society General Meeting, Denver, CO, USA, 26-30 July 2015; pp. 1-5.

(C) 2019 by the authors. Licensee MDPI, Basel, Switzerland. This article is an open access article distributed under the terms and conditions of the Creative Commons Attribution (CC BY) license (http:/ / creativecommons.org/licenses/by/4.0/). 